\title{
The bowfin genome illuminates the developmental evolution of ray-finned fishes
}

\author{
Andrew W. Thompson ${ }^{1,2}$, M. Brent Hawkins $\mathbb{1}^{3,4,5,6}$, Elise Parey $\mathbb{1}^{7}$, Dustin J. Wcisel ${ }^{8}$, Tatsuya Ota ${ }^{9}$, \\ Kazuhiko Kawasaki ${ }^{10}$, Emily Funk ${ }^{11,21}$, Mauricio Losilla ${ }^{11,2}$, Olivia E. Fitch ${ }^{11,2}$, Qiaowei Pan ${ }^{12}$, \\ Romain Feron ${ }^{12,13}$, Alexandra Louis ${ }^{7}$, Jérôme Montfort ${ }^{14}$, Marine Milhes ${ }^{15}$, Brett L. Racicot', \\ Kevin L. Childs ${ }^{16}$, Quenton Fontenot ${ }^{17}$, Allyse Ferrara17, Solomon R. David ${ }^{17}{ }^{17}$ Amy R. McCune ${ }^{11}$, \\ Alex Dornburg ${ }^{18}$, Jeffrey A. Yoder ${ }^{8,19,20}$, Yann Guiguen ${ }^{14}{ }^{14}$, Hugues Roest Crollius $\mathbb{D}^{7}$, Camille Berthelot ${ }^{7}$,

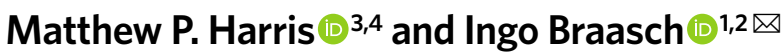

\begin{abstract}
The bowfin (Amia calva) is a ray-finned fish that possesses a unique suite of ancestral and derived phenotypes, which are key to understanding vertebrate evolution. The phylogenetic position of bowfin as a representative of neopterygian fishes, its archetypical body plan and its unduplicated and slowly evolving genome make bowfin a central species for the genomic exploration of ray-finned fishes. Here we present a chromosome-level genome assembly for bowfin that enables gene-order analyses, settling long-debated neopterygian phylogenetic relationships. We examine chromatin accessibility and gene expression through bowfin development to investigate the evolution of immune, scale, respiratory and fin skeletal systems and identify hundreds of gene-regulatory loci conserved across vertebrates. These resources connect developmental evolution among bony fishes, further highlighting the bowfin's importance for illuminating vertebrate biology and diversity in the genomic era.
\end{abstract}

T he monotypic bowfin, A. calva (Linnaeus, 1776), is a textbook example in comparative anatomy for its prototypical fish body plan and key phylogenetic position ${ }^{1,2}$. Bowfin biology thus sheds light on the evolution and development of ray-finned fishes and bony vertebrates in general. Ray-finned fishes constitute the most diverse vertebrate lineage with $>30,000$ living species, of which $>96 \%$ belong to the teleost fishes (Teleostei) ${ }^{3}$. The bowfin (Amiiformes) and seven gar species (Lepisosteiformes) represent the extant Holostei, the sister lineage of teleost fishes, together comprising the Neopterygii ${ }^{4-8}$. These eight holosteans, however, capture just a minor fraction of this once speciose lineage. The fossil record shows that the biodiversity of holosteans is highly underappreciated, as they were much more abundant in the past and as species rich as stem teleosts?.

With a multitude of teleost and a few non-teleost species, including spotted gar (Lepisosteus oculatus), sequenced ${ }^{7,10-13}$, the bowfin represents the last major neopterygian fish lineage remaining for detailed genomic and developmental exploration. Comparing bowfin and gar covers the maximum holostean genomic diversity available to study, and, due to their early divergence during holostean evolution $>250$ million years $\mathrm{ago}^{8}$, also spans the vast majority of holosteans to ever have existed ${ }^{9}$.
The bowfin is an important outgroup to investigate teleost evolution and development. The common ancestor of extant teleosts underwent the teleost whole-genome duplication (TGD), resulting in a sizeable fraction of often functionally divergent gene duplicates in living teleosts ${ }^{14,15}$. Genomic comparisons of teleosts with tetrapods such as humans are thus challenging, for example, in biomedical studies leveraging teleost models such as zebrafish, medaka, killifish or cavefish ${ }^{1}$. Bowfin and gar diverged from teleosts before the TGD and thus maintained more one-to-one gene relations with tetrapods ${ }^{7}$ and also have slower rates of molecular sequence evolution than teleosts ${ }^{7,16}$. Thus, the genome of the gar has been used as an intermediate steppingstone or 'bridge' for identifying hidden orthologies of genetic elements between teleosts and tetrapods ${ }^{7}$. By adding the bowfin genome, the 'holostean bridge' will capture more genomic diversity across the ray-finned tree of life and resolve critical questions about vertebrate evolution for which analyzing only one holostean representative is insufficient.

Importantly, within Neopterygii, the relations of bowfin, gars and teleosts have been matter of a long-standing, controversial debate (for example, refs. ${ }^{17-19}$ ) with two main proposed alternative scenarios. Bowfin has been grouped with gars in the Holostei clade with strong phylogenomic support (for example, refs. ${ }^{4-8,12}$ ), but,

\footnotetext{
'Department of Integrative Biology, Michigan State University, East Lansing, MI, USA. ${ }^{2}$ Ecology, Evolution \& Behavior Program, Michigan State University, East Lansing, MI, USA. 'Department of Genetics, Harvard Medical School, Boston, MA, USA. ${ }^{4}$ Department of Orthopedic Research, Boston Children's Hospital, Boston, MA, USA. ${ }^{5}$ Department of Organismic and Evolutionary Biology, Harvard University, Cambridge, MA, USA. ${ }^{6}$ Museum of Comparative Zoology, Harvard University, Cambridge, MA, USA. Institut de Biologie de I'ENS (IBENS), Département de Biologie, École Normale Supérieure, CNRS, INSERM, Université PSL, Paris, France. ${ }^{8}$ Department of Molecular Biomedical Sciences, NC State University, Raleigh, NC, USA. ${ }^{\circ}$ Department of Evolutionary Studies of Biosystems, SOKENDAI (the Graduate University for Advanced Studies), Hayama, Japan. ${ }^{10}$ Department of Anthropology, Pennsylvania State University, University Park, PA, USA. "Department of Ecology and Evolutionary Biology, Cornell University, Ithaca, NY, USA. ${ }^{2}$ Department of Ecology and Evolution, University of Lausanne, Lausanne, Switzerland. ${ }^{13}$ Swiss Institute of Bioinformatics, Lausanne, Switzerland. ${ }^{14}$ INRAE, LPGP, Rennes, France. ${ }^{15} \mathrm{GeT}$-PlaGe, INRAE, Genotoul, Castanet-Tolosan, France. ${ }^{16}$ Department of Plant Biology, Michigan State University, East Lansing, MI, USA. ${ }^{17}$ Department of Biological Sciences, Nicholls State University, Thibodaux, LA, USA. ${ }^{18}$ Department of Bioinformatics and Genomics, University of North Carolina at Charlotte, Charlotte, NC, USA. ${ }^{19} \mathrm{Comparative} \mathrm{Medicine} \mathrm{Institute,} \mathrm{NC} \mathrm{State} \mathrm{University,} \mathrm{Raleigh,} \mathrm{NC,} \mathrm{USA.}{ }^{20} \mathrm{Center}$ for Human Health and the Environment, NC State

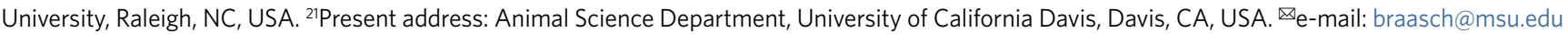


due to similarities in morphology, bowfin has also been historically grouped together with teleosts as Halecostomi (for example, refs. $\left.{ }^{17-20}\right)$. This Halecostomi scenario could further reconcile similarities of bowfin and teleost karyotypes ${ }^{21}$. A chromosomal bowfin genome assembly is thus essential to test these two alternative scenarios and to develop a comprehensive evolutionary framework for understanding phenotypic evolution among ray-finned fishes.

Bowfin's unique developmental, morphological, immunological and behavioral phenotypes, including pronounced sexual dimorphism $^{2}$, an evolutionarily informative immune system ${ }^{22}$, a derived type of dermal scales ${ }^{23}$, air breathing with a respiratory gas blad$\mathrm{der}^{24,25}$, and a largely ancestral fin skeleton ${ }^{1,20}$, are ripe for study with genomic sequence information. Here, we report a chromosome-level genome assembly that allows us to finally settle bowfin's phylogenetic position and to investigate chromosomal evolution, key gene families and gene-regulatory regions, as well as developmental processes in bowfin. We increase the utility of holostean genomic resources by characterizing chromatin accessibility and gene expression through bowfin development, enabling comparisons of non-coding regulatory regions across model and non-model fishes and tetrapods. The inclusion of the bowfin genomic landscape permits translation of genetic and genomic changes underlying vertebrate evolution and its regulation.

\section{Results}

The bowfin genome. The bowfin genome was sequenced from a single adult phenotypic male. A de novo genome assembly was constructed with Meraculous ${ }^{26}$ and further scaffolded with $\mathrm{Chicago}^{27}$ and $\mathrm{Hi}-\mathrm{C}$ approaches ${ }^{28}$ using the HiRise software pipeline ${ }^{27}$ (Supplementary Figs. 1 and 2). The final assembly $\left(N_{50}=41.2 \mathrm{Mb}\right)$ consists of 1,958 scaffolds including 23 pseudochromosomes that contain $99 \%$ of the assembly (Extended Data Fig. 1 and Supplementary Table 1) and match bowfin's chromosome number ${ }^{21,29}$, consistent with a chromosome-level genome assembly. The final assembly (AmiCal1, National Center for Biotechnology Information (NCBI) accession PESF00000000; Supplementary Tables 1 and 2) is largely complete as reported by multiple core eukaryotic genes mapping approach (CEGMA) and benchmarking universal single-copy orthologs (BUSCO) scores (Supplementary Table 3).

The bowfin genome consists of $22.1 \%$ repeats, very similar to that of spotted gar (22.8\%). However, there are clear differences between bowfin and gar in the distribution of individual transposable element (TE) types (Supplementary Table 4) and the evolutionary history of repeat amplification. Bowfin shows a single peak of TE activity (Kimura distance of 4), while spotted gar has two older TE bursts (Kimura distances of 7-8 and 25 (ref. ${ }^{7}$ )) (Supplementary Fig. 3).

Using transcriptomic evidence from ten adult tissues ${ }^{7,30}$, we generated a MAKER ${ }^{31}$ genome annotation reporting 21,948 protein-coding genes, very similar to that for spotted gar $(21,443$ genes $)^{7}$. OrthoFinder ${ }^{32}$ predicted orthologies for $86.6 \%$ of these genes to 11 other vertebrates (Supplementary Tables 5 and 6). See Supplementary Notes 1 and 2 for genome assembly and annotation details.

Despite pronounced sexual dimorphism in adult behavior and color patterning in bowfin (Fig. 1a, Supplementary Fig. 4a,b and Supplementary Note 3), its karyotype does not show any obvious cytogenetic differentiation of sex chromosomes ${ }^{21,29}$. Here, pool-sequencing (Pool-seq) strategies that contrast 30 mature phenotypic males with 30 mature phenotypic females using both reference genome-based and genome-free approaches did not reveal any genomic region exhibiting sex differentiation (Supplementary Fig. 4c-f, Supplementary Tables 7 and 8 and Supplementary Note 3), similar to previous findings for spotted $\operatorname{gar}^{33}$. Sex chromosomes and genetic sex-determination mechanisms thus remain elusive in holosteans if they exist.
Genome organization and gene order support the monophyly of holostean fishes. Cytogenetic comparisons suggest that the bowfin karyotype is more similar to those of teleosts than those of gars ${ }^{21}$. While gar and chicken retained micro-chromosomes from the bony vertebrate ancestor ${ }^{7,34,35}$, karyotype analyses provide no evidence for micro-chromosomes in bowfin ${ }^{21,29}$. To the best of our knowledge, true micro-chromosomes have also not been discovered in teleosts. This shared absence of micro-chromosomes could imply a closer relationship of bowfin and teleosts (Halecostomi) opposed to the monophyly of bowfin and gars (Holostei). We previously showed that the teleost ancestor experienced micro-chromosome fusions after its divergence from gar but before the TGD ${ }^{7}$. We thus investigated whether these teleost micro-chromosome fusions were shared with bowfin (Fig. 1 and Extended Data Fig. 2a-c). By orthology to gar micro-chromosomes conserved from the bony vertebrate ancestor ${ }^{7}$, we identified bowfin and teleost chromosomes corresponding to fused ancestral micro-chromosomes. We found three such micro-chromosome fusions for bowfin and five for the teleost medaka, with none shared between them (Fig. 1c,d). These fusions were also not shared with micro-chromosome fusions in the derived genome of bichir (Supplementary Fig. 5), representing the most basally diverging extant ray-finned fish lineage ${ }^{12}$. We conclude that bowfin, teleosts and bichir independently fused different sets of micro-chromosomes. The karyotypic similarities of bowfin and teleosts are the result of convergent evolution rather than due to a common origin and thus do not support the Halecostomi scenario.

We identified four chromosome pairs with one-to-one orthology between bowfin and chicken, while there are 14 one-to-one pairs between gar and chicken ${ }^{7}$ (Extended Data Fig. 2d,e). Thus, the bowfin karyotype is more derived than that of gar. However, in support of holostean monophyly, chicken chromosomes 13 and 23 correspond to a fusion chromosome in both bowfin and gar (Extended Data Fig. 2f), a rearrangement not found in teleosts or bichir (Supplementary Fig. 5).

We investigated gene-order evolution as a new line of evidence to illuminate bowfin's phylogenetic position. Genomic rearrangements (inversions, transpositions, translocations) introduce gene-order differences across species over evolutionary time but are much rarer than nucleotide substitutions and therefore useful for reconstructing phylogenies ${ }^{36}$. Using rearrangement distances, we reconstructed a phylogeny of bowfin, gar and ten teleosts, with the addition of two tetrapod outgroups, and leveraged all 3,223 marker genes that are present in exactly one copy in non-teleost genomes and in one or two copies in TGD-derived teleost genomes. We then estimated pairwise evolutionary distances between species using a normalized breakpoint distance (Supplementary Fig. 6) and constructed rearrangement-based neighbor-joining (NJ), minimum evolution and unweighted pair group method with arithmetic mean (UPGMA) species trees that all support holostean monophyly with high confidence (Fig. 1e). Focusing on all gene adjacencies acquired since the neopterygian ancestor (that is, gene adjacencies found in gar, bowfin and/or teleosts but not in outgroups), we positioned gains and losses of these adjacencies on the two alternative phylogenies using the Dollo algorithm, assuming that a gene adjacency is gained only once but can be lost numerous times independently ${ }^{37}$. The Holostei scenario required a significantly lower number of evolutionary changes (760 versus 1,024 ; Kishino-Hasegawa $(\mathrm{KH})$ test, $P$ value $=0.028)$, rejecting the Halecostomi scenario (Fig. 1f).

Phylogenomic analyses of OrthoFinder ${ }^{32}$-generated protein alignments from 2,079 single-copy genes in 12 vertebrate species using maximum-likelihood and Bayesian approaches, as well a species tree generated from 7,532 OrthoFinder gene trees using $\mathrm{STAG}^{38}$, provide additional, strong evidence for holostean monophyly (Supplementary Note 4 and Supplementary Fig. 7). Thus, gene order, in agreement with our sequence-based analyses and published phylogenomic studies using coding and non-coding 


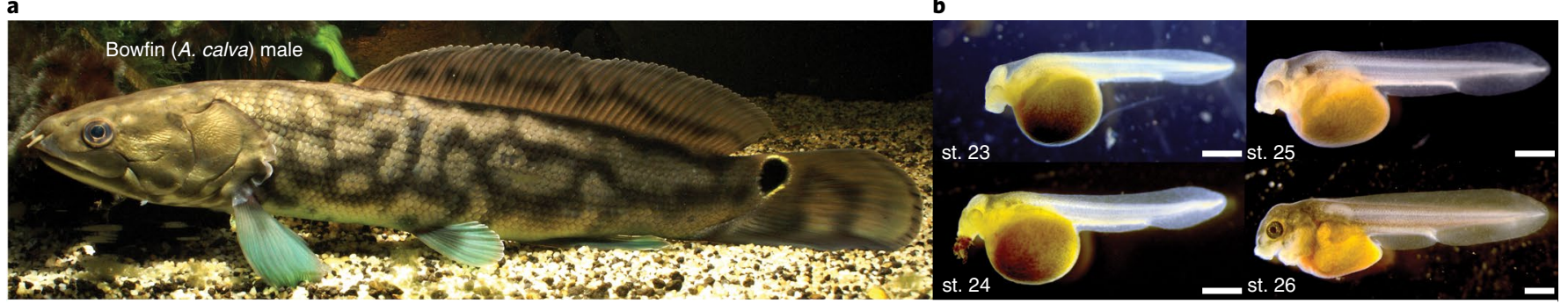

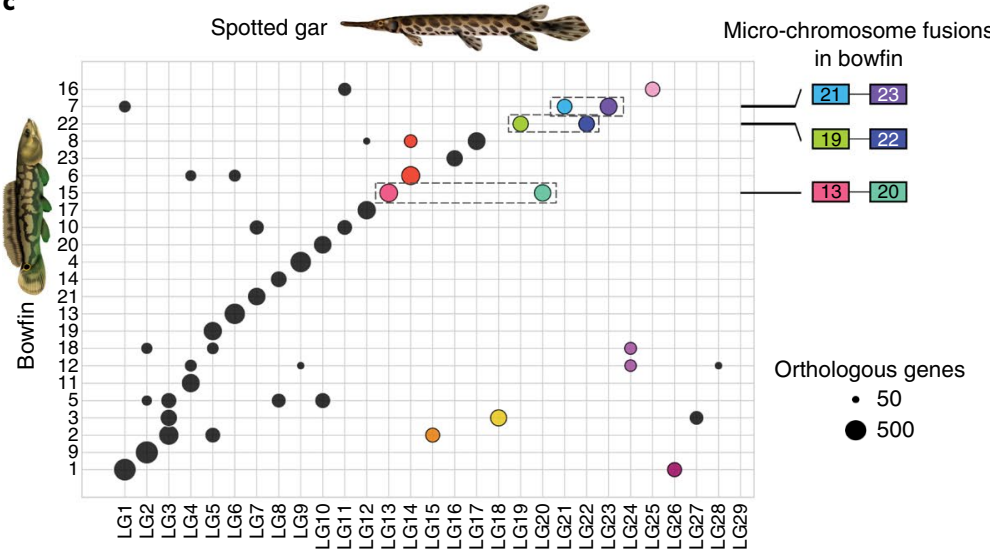

d

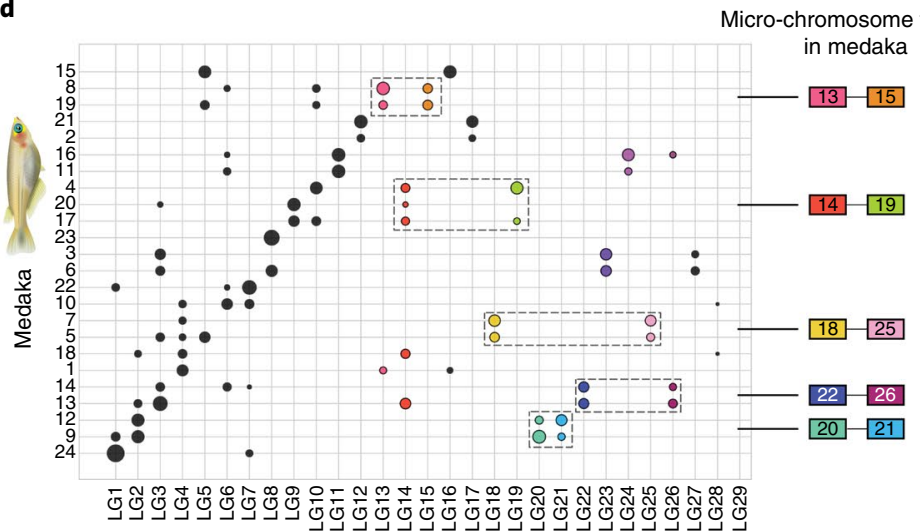

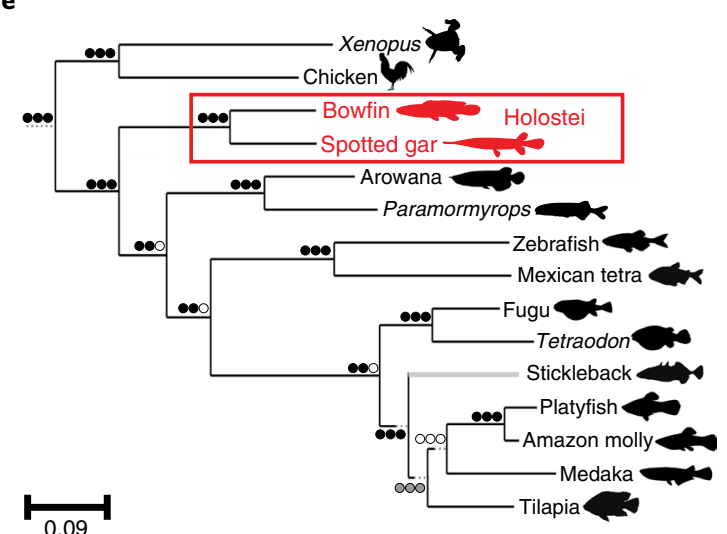

f

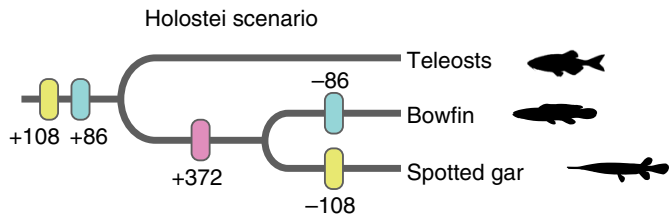

Adjacency gains + losses: 760

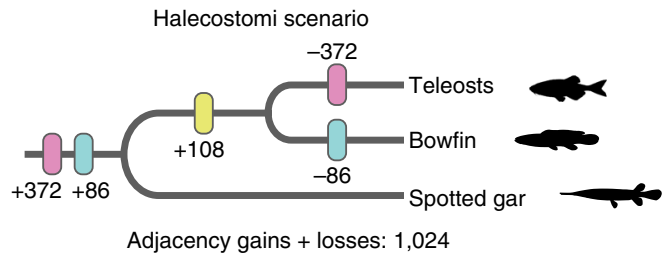

Fig. 1 | Bowfin and the evolution of neopterygian genome organization. a, Adult male bowfin. b, Bowfin stages (st.) 23-26 (ref. ${ }^{53}$ ), covering critical phases of pectoral fin and gas bladder development (representative of $n=20$ individuals per stage). Scale bar, $1 \mathrm{~mm}$. c, Orthologies between bowfin and gar chromosomes and inferred bowfin micro-chromosome fusions for bowfin. $\mathbf{d}$, Orthologies between medaka and gar chromosomes and inferred medaka micro-chromosome fusions. Circles, number of orthologous genes shared by bowfin and gar and/or medaka chromosomes (if in excess compared to random expectations). Ancestral micro-chromosomes are highlighted with colors; micro-chromosome fusions are indicated by dotted boxes. Colored boxes refer to the gar chromosome number. Micro-chromosome fusions differ between $\mathbf{c}$ and $\mathbf{d}$ : for example, medaka 9 and 12 result from a pre-TGD fusion of ancestral chromosomes orthologous to gar micro-chromosomes LG20 and LG21 and macro-chromosome LG2, followed by TGD duplication of the fusion chromosome (d). Bowfin 15 is a fusion of ancestral micro-chromosomes orthologous to gar LG13 and LG20; bowfin 7 is a fusion of ancestral chromosomes orthologous to gar LG1 and micro-chromosomes LG21 and LG23 (c). e, NJ phylogeny based on gene-order divergence built using a normalized breakpoint distance. Circles, bootstrap support for NJ, FastME and UPGMA analyses; black, 100\%; gray $\geq 70 \%$; white $<70 \%$. The Holostei clade is strongly supported (red box). Note that the location of the stickleback branch (gray) is in disagreement with the consensus phylogeny ${ }^{4,8}$ (but see ref. ${ }^{85}$ ). f, Dollo parsimony applied to gains or losses of local gene adjacencies in Holostei (top) and Halecostomi (bottom) scenarios. Adjacencies shared by bowfin and gar only are in pink, those shared by bowfin and teleosts only are in yellow, and those shared by gar and teleosts only are in blue.

markers ${ }^{4-8,12}$, strongly supports holostean monophyly regardless of methodology.

While the bowfin karyotype is more derived than that of gar at a gross chromosomal level, this is not reflected in local gene order, for which bowfin and gar present similar levels of species-specific rearrangements rates (Supplementary Note 5). In agreement with the slow rate of genomic sequence evolution in holosteans ${ }^{7,16}$, gene-order rearrangement rates in bowfin are significantly lower than those in teleosts (Supplementary Note 5).

Characterizing the holostean immunogenome. Our previous analyses of spotted gar immune genes revealed shared characteristics with those of both teleosts and tetrapods but left important aspects of the ray-finned fish immunogenome unresolved ${ }^{7,39}$. For a 
comprehensive understanding of holostean immune genes, we surveyed the bowfin genome and a newly generated bowfin immune tissue transcriptome (Supplementary Note 6).

The human major histocompatibility complex (MHC) is a cluster of $>200$ genes, broadly characterized as class I, II and III genes, the products of which play various roles in antigen processing, antigen presentation and inflammation ${ }^{40}$. Class I and class II genes are tightly linked on one chromosome in cartilaginous fishes and tetrapods (for example, on human chromosome 6, Fig. 2) ${ }^{41}$. By contrast, teleost class I and class II genes are not linked, and class III genes are scattered throughout teleost genomes ${ }^{42}$. The ancestral organization of the ray-finned $\mathrm{MHC}$ has remained unresolved because the gar $\mathrm{MHC}$ is highly fragmented in the reference assembly ${ }^{7}$. The bowfin, by contrast, has a cluster on pseudochromosome 14 that contains the majority of class I, II and III genes (Fig. 2, Supplementary Figs. 8 and 9, Supplementary Tables 9-11 and Supplementary Note 6) and that is not the result of a recent chromosome fusion (Fig. 1c and Supplementary Fig. 8). Thus, the overall organization of MHC regions in holostean and ray-finned fish ancestors was similar to that in tetrapods and cartilaginous fishes. Furthermore, the loss of linkage of teleost class I, II and III genes therefore occurred after divergence from holosteans and is associated with differential gene loss from TGD-duplicated chromosomes, exemplified by $\mathrm{MHC}$ regions on zebrafish chromosomes 19 and 16 (Fig. 2 and Supplementary Fig. 8).

Antigen-recognition receptors of the adaptive immune system, that is, immunoglobulin (Ig) and T cell receptor (TCR), have been identified in all jawed vertebrate lineages but can differ in their genomic structure and organization ${ }^{43}$. The bowfin genome contains all three canonical TCR loci and, remarkably, encodes not only antibody classes IgM and IgD but also IgT (IgZ in zebrafish) (Extended Data Fig. 3, Supplementary Figs. 10-12, Supplementary Tables 12 14 and Supplementary Note 6), which has been considered teleost specific ${ }^{44}$. We then used the bowfin gene encoding IgT, which is confirmed by transcriptomic analyses ${ }^{45}$, to identify a previously unknown ${ }^{7}$ IgT ortholog in spotted gar. Thus, IgT-like antibodies date back to the neopterygian ancestor (Supplementary Note 6$)^{45}$.

Toll-like receptors (TLRs) provide initial immune responses to infection ${ }^{46}$. The bowfin possesses 20 TLR genes, twice the number of human TLR genes ${ }^{47}$, more than the 16 functional spotted gar TLR genes ${ }^{7,39}$ and almost on par with the $20+$ teleost TLR genes ${ }^{48}$ (Supplementary Fig. 13, Supplementary Table 15 and Supplementary Note 6). TLR complexity is therefore a more general pattern among neopterygians, and the TGD is not the sole evolutionary mechanism leading to large TLR gene numbers in ray-finned fishes. In summary, the holostean immunogenome shows an overall complexity and diversity comparable to those of teleosts, with a chromosomal organization resembling tetrapods and more distant vertebrate lineages.

Biomineralization genes and the formation of ray-finned fish scales. While bowfin and gar both possess enamel-covered teeth, they prominently differ in scale biomineralization. Gars have ganoid scales, representing an ancestral actinopterygian scale type covered with a thick layer of hypermineralized ganoin. The bowfin, by contrast, has thin, flexible elasmoid scales that secondarily lost the thick bony plate and ganoin ${ }^{17,23}$. Teleosts have neither tooth enamel nor scale ganoin. It is thus expected that genes involved in scale formation differ among neopterygians.
The secretory calcium-binding phosphoprotein (SCPP) gene family, generated by complex successive gene duplications during bony vertebrate evolution, encodes proteins involved in biomineralization $^{49}$. We identified 22 SCPP genes in bowfin, 21 of which form two large genomic clusters arranged similarly to those in gar (Fig. 3, Supplementary Data 1 and Supplementary Note 7). Similar to $\operatorname{gar}^{7,50,51}$, the bowfin possesses ganoin-forming SCPP genes enamelin (enam), ameloblastin ( $a m b n$ ) and scpp5 (Fig. 3). Their involvement in the formation of both tooth enamel and scale ganoin in gar supports the idea that these two mineralized structures evolved from a common genetic program ${ }^{7,50,51}$. As bowfin lacks ganoin, this suggests that gene-regulatory shifts in enam, ambn and scpp5 have occurred in the bowfin lineage that silenced their expression during scale development but not dental enamel formation.

The total number of bowfin SCPP genes (22) is considerably smaller than that of gar, which has the largest known SCPP gene repertoire among vertebrates $(38)^{7,49}$. All 22 bowfin SCPP genes have a gar ortholog. By contrast, orthologs of 16 gar SCPP genes could not be found in bowfin, nine of which are located in a cluster on gar chromosome LG2 syntenic to a reduced gene cluster on bowfin pseudochromosome 9 (Fig. 3). Expression of most gar LG2 SCPP genes was weak or undetectable in tooth germs but strong in scale-forming $\operatorname{skin}^{7}$. This implies that the SCPP clusters on gar LG2 and bowfin pseudochromosome 9 are particularly involved in scale formation, a hypothesis further supported by teleost SCPP genes (Supplementary Fig. 14 and Supplementary Note 7). The orthologous zebrafish SCPP genes are located on TGD-duplicated clusters on chromosomes 5 and 10 (ref. ${ }^{7}$ ) and are expressed during zebrafish skin development (Supplementary Table 16 and Supplementary Note 7). Furthermore, these clusters have been reduced to only one gene $(s c p p 8)$ in the scaleless channel catfish (Fig. 3). They are also highly reduced in scale-reduced sturgeon and paddlefish ${ }^{13}$ (Supplementary Note 7). These multiple lines of evidence suggest that SCPP genes with major roles in scale formation are clustered in a genomic region that is expanded in the ganoid gar on LG2 and likely secondarily reduced in bowfin on pseudochromosome 9, corresponding to the formation of their modified elasmoid scales. We thus hypothesize that reduced biomineralization of the bowfin scale is attributed to both changes in gene regulation and the loss of specific SCPP genes.

\section{Chromatin profiling through bowfin development connects gene regulation across vertebrate morphologies. To increase the power} and utility of the holostean genome for comparative studies on vertebrate gene regulation, we used the assay for transposase-accessible chromatin with sequencing (ATAC-seq) s2 $^{52}$ to generate an atlas of open chromatin regions (OCRs) from seven developmental stages of wild-caught bowfin embryos and larvae, from before the conserved phylotypic stage to the end of the described larval development ${ }^{53}$ (Supplementary Fig. 15 and Supplementary Note 8). We identified a total of 172,276 OCRs, of which $81.8 \%(140,902)$ were non-coding OCRs (ncOCRs) and annotated their genomic feature location and nearest gene with $\mathrm{HOMER}^{54}$ (Supplementary Tables 17-19). About $70 \%$ of OCRs and ncOCRs were identified in at least two stages; 33,239 OCRs and 21,636 ncOCRs were found in all seven stages (Supplementary Tables 17 and 18). Using whole-genome alignments (WGAs) generated with Progressive Cactus ${ }^{55}$, we showed that more than $50 \%$ of bowfin ncOCRs were conserved in gar, and 3,844 core bowfin ncOCRs were conserved in gar, zebrafish, mouse

Fig. 2 | The bowfin MHC. a, The bowfin MHC on pseudochromosome 14 contains class I, class II and class III genes with orthologous relationships to the human $\mathrm{MHC}$ on chromosome 6 and zebrafish chromosomes 19, 16 and 15. Boxes represent genes or gene clusters of related sequences; gene placement is not to scale. Only those genes with bowfin orthologs are shown in human and zebrafish. Pseudogenes of non-MHC genes and RNA-coding genes are not included. b, Detailed lists of genes within each human leukocyte antigen (HLA) gene cluster in human (for example, William W. Ballard) indicated in a are provided. Asterisks indicate MHC or MHC-related pseudogenes. See also Supplementary Table 9. 
a

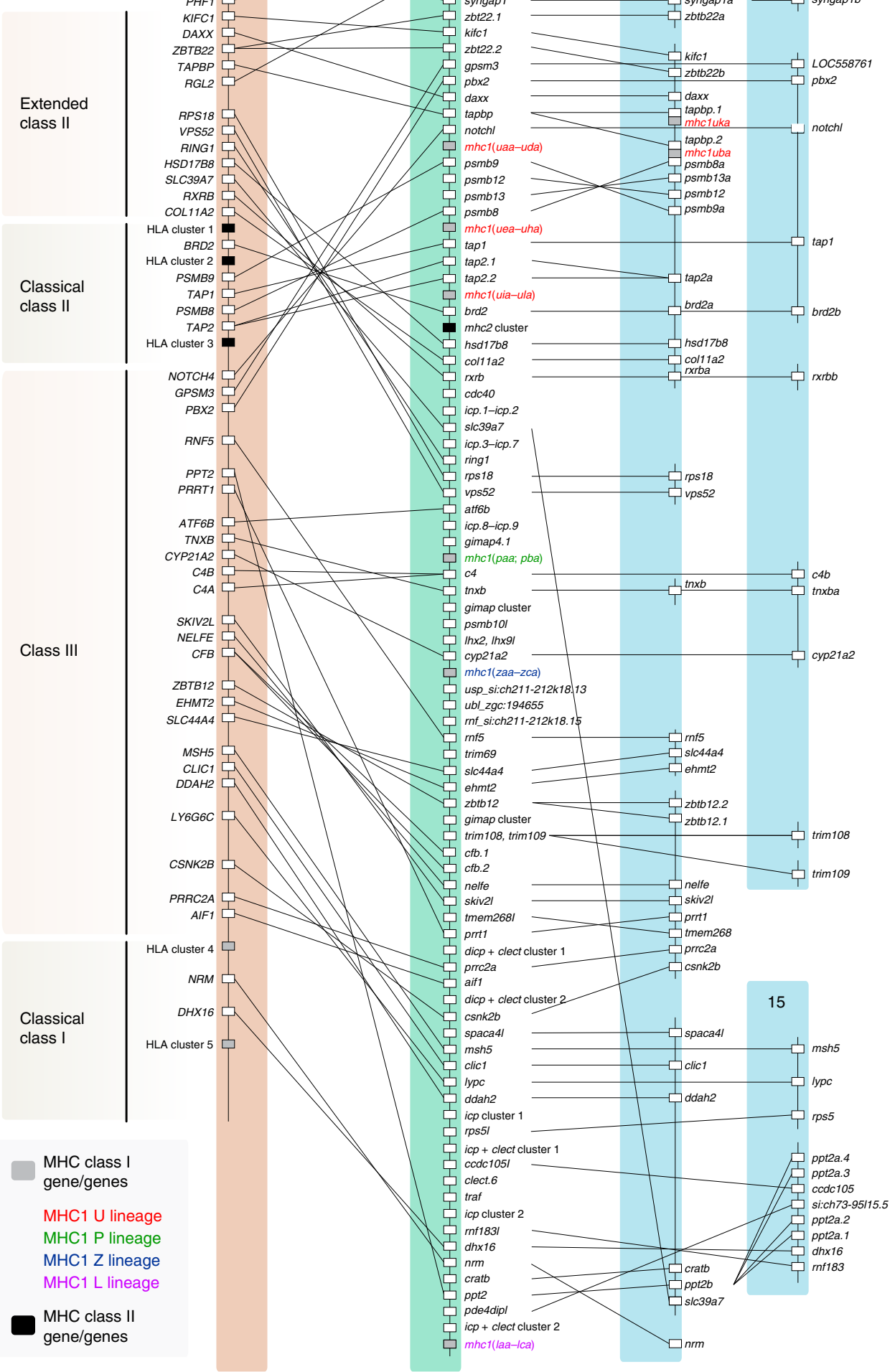

b

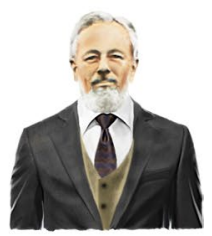

Human

HLA clusters

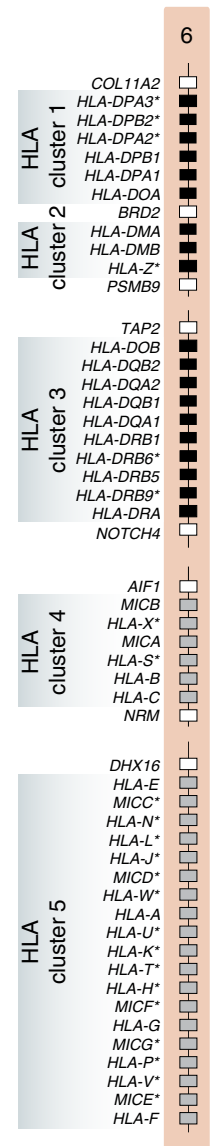



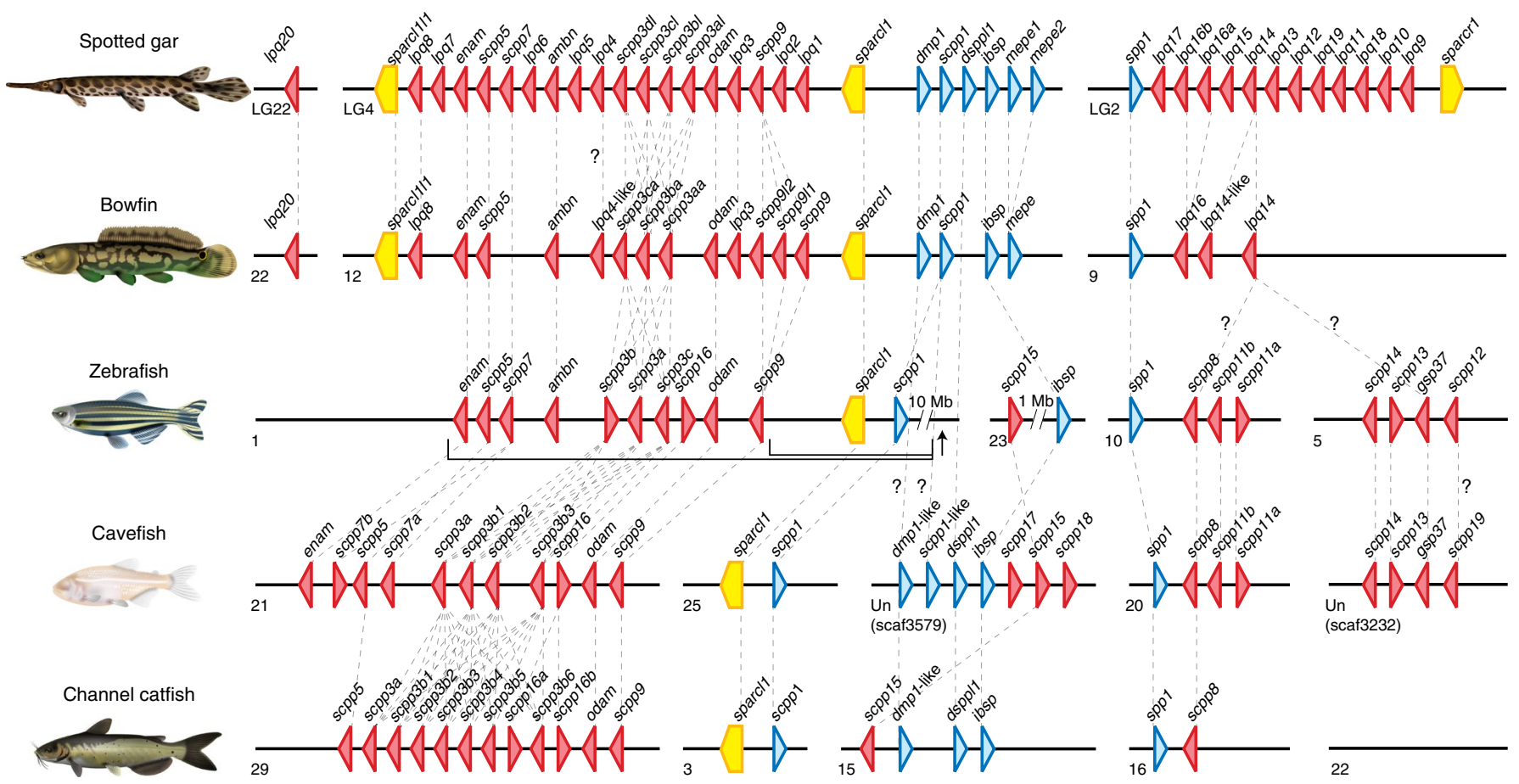

22

Fig. 3 Organization of biomineralization genes in neopterygian fishes. Genomic arrangements of SCPP genes and secreted protein acidic and rich in cysteine (SPARC)-family genes in the genomes of spotted gar, bowfin and the teleosts zebrafish, Mexican tetra (cavefish) and channel catfish. The direction of triangles and pentagons indicates the transcriptional direction of each gene, encoding P/Q-rich SCPPs (red), acidic SCPPs (blue) and SPARC-family proteins (yellow). Orthologs are connected by dashed lines; unresolved orthologies are indicated with a '?'. Intervening non-SCPP genes are not shown. In the zebrafish genome, a cluster of sparcl1 and scpp1 is separated by $10 \mathrm{Mb}$ from the other genes encoding P/Q-rich SCPPs (arrow). Two cavefish SCPP gene clusters are located on unplaced scaffolds. See Supplementary Data 1 and Source Data for SCPP gene predictions, locations and gene IDs.

and human (Supplementary Fig. 16 and Supplementary Table 20). Adding RNA-seq transcriptomes from the same stages, our dataset provides a rich resource for exploring the correlation between chromatin accessibility of putative gene-regulatory elements and gene expression. This is exemplified by bowfin ncOCRs overlapping key enhancers active during the development of a variety of vertebrate systems such as the brain ${ }^{56}$, heart ${ }^{57}$, anterior and posterior fin or limb ${ }^{58-60}$ and lungs ${ }^{60,61}$ (Fig. 4). Using Hi-C contact maps from bowfin blood cells, we further identify a bowfin ncOCR at the location of an evolutionary conserved hemoglobin gene enhancer ${ }^{62}$, embedded within a topologically associating domain (TAD) that shows conserved synteny (Extended Data Fig. 1c-f) with tetrapod hemoglobin-region TADs ${ }^{63,64}$.

Evolutionary conserved elements such as conserved non-coding elements (CNEs) and ultraconserved elements (UCEs) (as defined in the Methods) are used to identify candidate enhancers and genetic loci for phylogenetic inference ${ }^{6,7}$. With our WGAs, we found that $31.9 \%$ of both gar-centric vertebrate $\mathrm{CNEs}^{7}(21,127$ of 66,182$)$ and $27.5 \%$ of bowfin UCEs ${ }^{6}$ (100 of 364) intersected with bowfin ncOCRs (Supplementary Table 21); thus, many CNEs or UCEs are likely active gene-regulatory elements during bowfin development, some of which are specific to different fish clades (Supplementary Table 21 and Supplementary Note 8). Overlap with bowfin ncOCR increases with CNE age, suggesting that ancestry of sequence conservation is a predictor of chromatin accessibility during bowfin development (Supplementary Table 21 and Supplementary Note 8). To further investigate the comparative efficacy of our ATAC-seq dataset, we used our WGAs to identify bowfin orthologs of experimentally confirmed mammalian VISTA enhancers ${ }^{56}$ (Supplementary Tables 22 and 23 and Supplementary Note 8). Orthologs of $60.2 \%$ of human VISTA enhancers (600 of 996) were found in bowfin, and over half (56.3\%, 338 of 600) overlapped with bowfin ncOCRs. By contrast, orthology for only 44.0\% (449 of 996) of human enhancers could be established in the zebrafish genome (Supplementary Table 22), illustrating the usefulness of the slowly evolving and 'unduplicated' bowfin genome to connect accessible, non-coding, regulatory regions from human to fish. Overlap with 2,261 ncOCRs from a mouse developmental single-nucleus ATAC-seq atlas ${ }^{65}$ (22.5\% of 10,035 mouse ncOCRs with bowfin orthology) established bowfin ncOCRs that likely function in a cell and/or tissue type-specific manner, for example, in the central nervous system, mesoderm, neural crest and many other cell types and tissues (Supplementary Table 24).

Currently, beyond zebrafish and medaka (for example, refs. ${ }^{66,67}$ ), there are limited gene-regulatory resources for fishes, rendering comparative analyses across species or whole-genome duplications difficult. Our bowfin ATAC-seq atlas provides a key platform to further connect gene-regulatory homology across vertebrates while strengthening the utility of non-teleost fishes for examining genome function in the context of long-standing questions about vertebrate evolution and development.

Deep homology of vertebrate air-filled organs at the gene-regulatory level. Ever since Owen and Darwin, the homology of vertebrate air-filled organs has been debated ${ }^{68}$ and is supported by adult gene expression similarities among various fish air bladders and tetrapod terrestrial lungs (for example, refs. ${ }^{12,69,70}$ ). Our previous genetic developmental investigations ${ }^{24,25}$ in bowfin identified several key developmental genes for respiratory gas bladder development including the $t b x 4$ transcription factor gene. Using our ATAC-seq atlas, we identified an intronic ncOCR within the bowfin $t b x 4$ gene (Fig. $4 \mathrm{f}$ ) that is orthologous to a $t b \times 4$ 'lung enhancer' important for embryonic lung budding in mammals ${ }^{60,61}$ (Extended Data Fig. 4a,b and Supplementary Note 9). Furthermore, we showed 

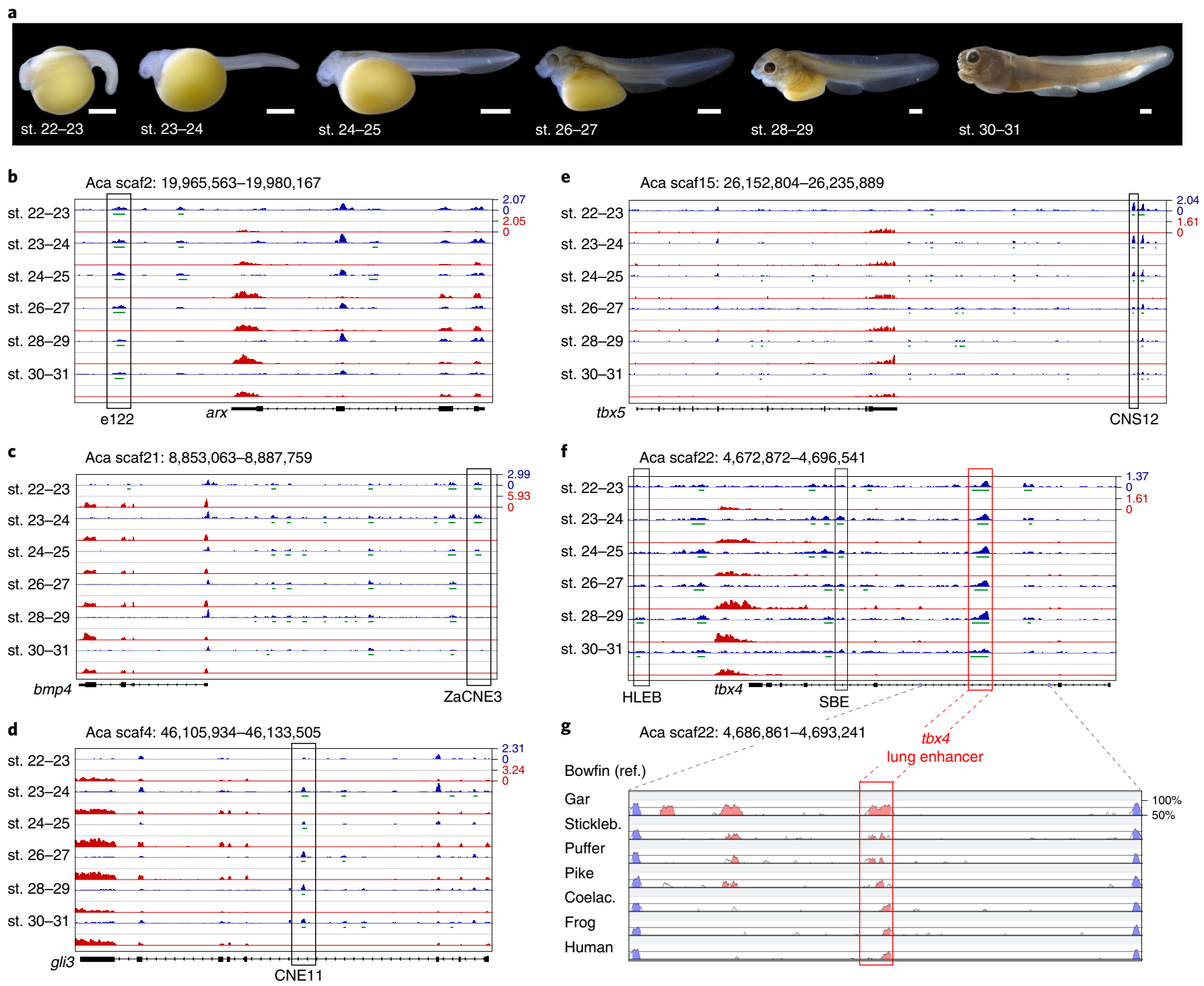

g Bowfin (ref.)

tbx4

lung enhancer

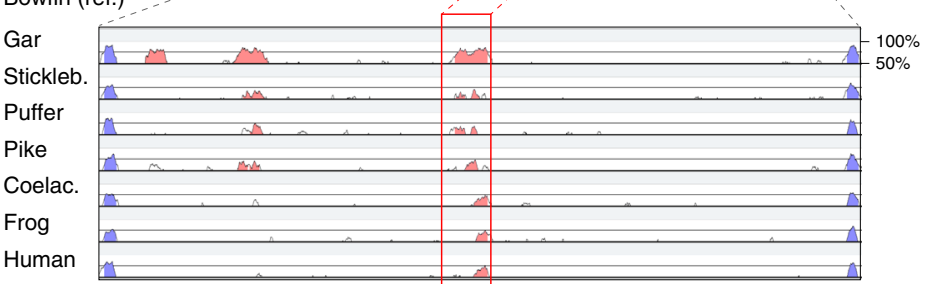

Fig. 4 | Chromatin and gene expression profiles during bowfin development. a, Developmental-stage series of bowfin ${ }^{53}$ (representative of $n=5$ individuals per stage). Scale bars, $1 \mathrm{~mm}$. b-f, ncOCRs overlapping with key developmental enhancers are indicated by black boxes. ATAC-seq profiles are in blue, MACS2-called OCRs are in green, mRNA-seq profiles are in red, and gene annotations are in black. ATAC-seq and RNA-seq data ranges are indicated for each panel. b, Forebrain enhancer e122 (ref. ${ }^{56}$ ) downstream of arx is consistently open. Aca, A. calva. c, Pan-cardiac heart enhancer ZaCNE3, conserved from tetrapods to fish ${ }^{57}$ and upstream of $b m p 4$, transitions from open to closed chromatin as development progresses. $\mathbf{d}$, Intragenic gli3 limb enhancer CNE11 (ref. ${ }^{58}$ ), connected by sequence orthology from tetrapods to teleosts through gar ${ }^{7}$ and bowfin, opens at stage 23 when pectoral fins are first visible ${ }^{53}$ and remains open during pectoral fin development. e, Pectoral fin tbx5 enhancer CNS12 (ref. ${ }^{59}$ ) is open during stages $22-27$ when pectoral fins develop. f, The tbx4 hindlimb enhancer HLEB ${ }^{60}$ is an ncOCR at stages $28-30$, shortly after the onset of pelvic fin development in stage 27 (ref. ${ }^{53}$ ). Another ncOCR is located on the tbx4 'lung enhancer', critical for lung development in mouse ${ }^{60,61}$, that is open throughout bowfin gas bladder budding and outgrowth stages (stages 24-27) when tbx4 is expressed in the bowfin gas bladder ${ }^{24}$. A putative swim bladder enhancer (SBE) ${ }^{86}$, conserved with teleosts (Extended Data Fig. 4), is also marked by open chromatin. $\mathbf{g}$, VISTA-SLAGAN ${ }^{87,88}$ conservation plot with bowfin as reference (ref.) shows sequence conservation of the tbx 4 lung enhancer from tetrapods to lobe-finned fish (coelacanth (coelac.)) and teleosts (stickleback (stickleb.), pufferfish, pike), demonstrating the power of the 'holostean bridge' to reveal deep homology of gene regulation of key vertebrate morphologies (Extended Data Fig. 4 and Supplementary Note 9).

sequence conservation with a previously uncharacterized, conserved intronic region in teleost fishes (Fig. $4 \mathrm{~g}$ ). Thus, the 'holostean bridge' establishes orthology of this important developmental enhancer from tetrapods to teleosts (Fig. 4g, Extended Data Fig. 4c and Supplementary Note 9). Importantly, the chromatin accessibility of the 'lung enhancer' activity in bowfin correlates with critical timing of $t b x 4$ expression during gas bladder development ${ }^{24}$ (Fig. $4 \mathrm{f}$ and Supplementary Note 8). These results support deep homology among bony vertebrate air-filled organs, that is, terrestrial lungs, holostean respiratory gas bladders and buoyancy-controlling swim bladders in teleosts (Supplementary Note 9) at the developmental, gene-regulatory level.

Evolutionary stasis of holostean Hox clusters and the enigmatic hoxD14 gene. Hox cluster genes play essential roles in patterning the primary body axis during animal development as well the proximo-distal and anterior-posterior axes of vertebrate appendages. As a result of the two early rounds of vertebrate genome duplication, non-teleost ray-finned fishes without additional genome duplications such as gar contain four Hox clusters, Hox $\mathrm{A}-\mathrm{D}^{7}$. 


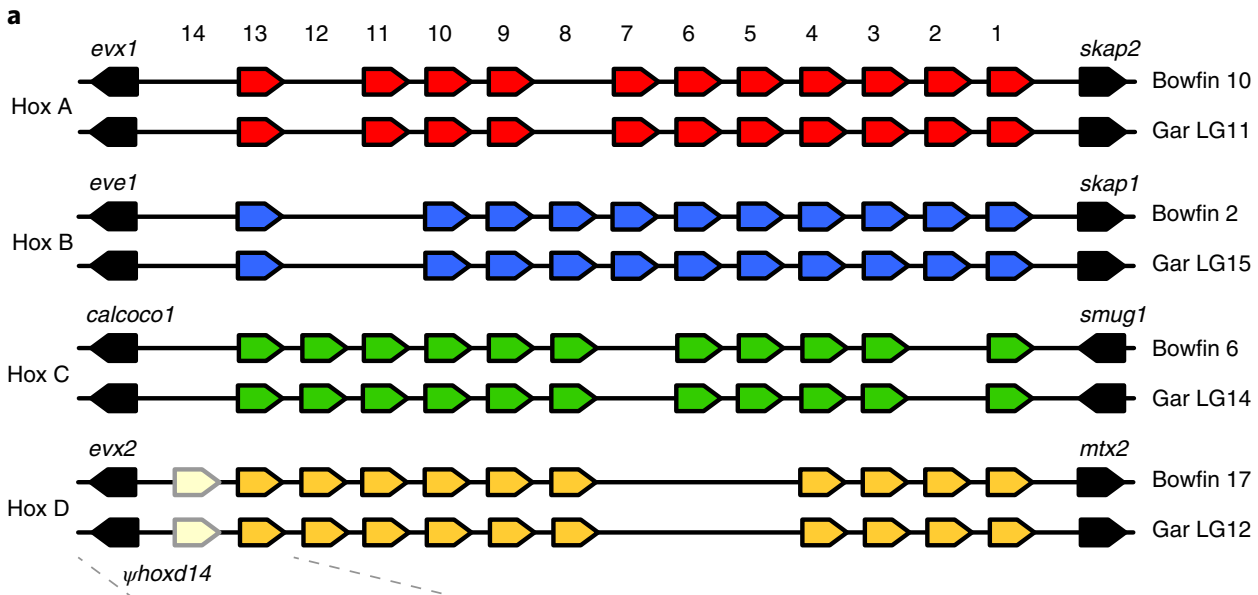

b

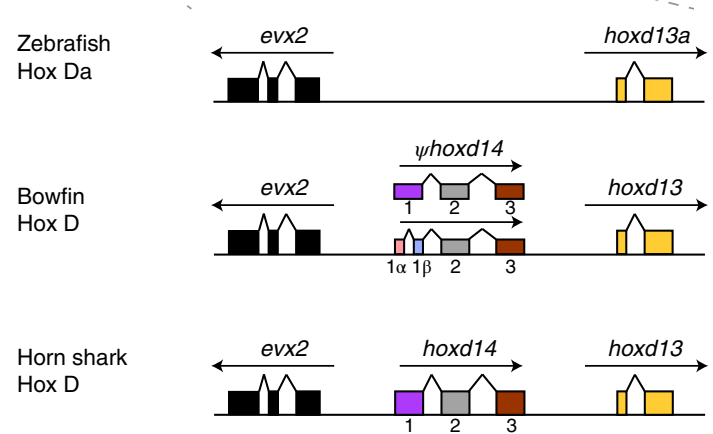

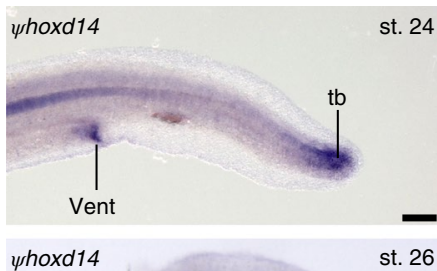

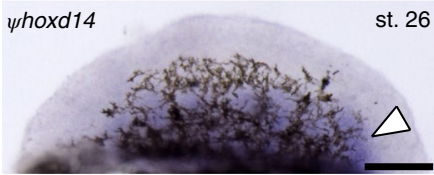

$\nabla$

Aca $\psi$ hoxd14 VSKYTCEVRRKGPINSK*QIAGL * LEFERNNFLTPKNFIKTERQVKLWFQNQRLKOKKFL Loc $\quad$ hoxd14 QGKR*IPYSKHLTAEL *EGIREE*FSPSRNS IELFAKA*SYRKAV* IWFQNQKQKRLHI * Psp hoxd14 QRKKRIPYSNHQRAELEKAFEKNRFLTPENRLNISSKLALTEROVKIWFQNQROKEKKLL Cmi hoxd14 QRKKRIPYSKLQITELEKAFENNRFLTPEIRLNISLKLGLTERQVKLWFQNQRQKEKKLL Hfr hoxd14 QRKKRVPYSKOQIAELEMAYENNRFLTPEVRLNISFKLGLTERQVKIWFQNQRQKEKKLL Sca hoxd14 QRKKRIPYSKQQITELEMAFENNRFLTPEVRLNISFKLGLTERQVKIWFQNQRQKEKKLL

Fig. 5 | Holostean Hox gene clusters and the bowfin hoxd14 pseudogene. a, Hox cluster organization in bowfin and spotted gar genomes. b, Relative position and orientation of genes encoding Hox14 paralogs in the Hox D cluster between evx2 and hoxd13 in zebrafish, bowfin and horn shark. We identified two alternative splice variants in bowfin. c, Whole-mount RNA in situ hybridization of hoxd14 pseudogene expression in the tailbud (tb) and vent at stage 24 and in the posterior pectoral fin mesenchyme at stage 26 (white arrowhead), observed consistently across $n=10$ samples. Scale bars are $125 \mu$ m. d, Multiple-sequence alignment of the HoxD14 homeodomain across species including bowfin (Aca), spotted gar (Loc), paddlefish (Psp, Polyodon spathula), elephant shark (Cmi, Callorhinchus milii), horn shark (Hfr, Heterodontus francisci) and small-spotted catshark (Sca, Scyliorhinus canicula). The black arrowhead indicates the conserved intra-domain splicing position, and underlining indicates the conserved WFQNQR motif.

Highlighting the evolutionary stasis of the holostean lineage, the bowfin genome harbors the same repertoire of 43 bona fide Hox cluster genes as spotted gar (Fig. 5a, Supplementary Data 2 and Supplementary Note 10), with only one gene lost since the bony vertebrate ancestor and none lost since the ray-finned and holostean fish ancestors ${ }^{7,11}$.

Evolution of the vertebrate Hox14 paralog group exhibits unique patterns of conservation, loss and pseudogenization. Members of this paralogy group are absent from tetrapods and teleosts but present in lamprey, cartilaginous fishes, paddlefish, sturgeon and $\operatorname{gar}^{7,11,71-73}$. Gar hoxd14 is a pseudogene without evidence for transcription $^{7}$ (Supplementary Note 10). We found sequence conservation of gar hoxd14 with the bowfin genome within the intergenic region between $e v x 2$ and hoxd13. A large number of bowfin pectoral fin bud RNA-seq reads (see below) aligned to this region, which also showed open chromatin status (Supplementary Fig. 17). We cloned two different hoxd14 transcripts from bowfin pectoral fin bud cDNA (Fig. 5b). RNA in situ hybridization revealed that bowfin hoxd14 is expressed in stage 26 pectoral fin buds in a posterior mesenchymal domain (Fig. 5c), similar to expression in paddlefish $^{73}$. Expression was also observed in the vent, similar to that in shark and lamprey ${ }^{71}$, and in the tailbud (Fig. 5c). Bowfin hoxd14 is likely a pseudogene, with numerous stop codons and nonsynonymous changes throughout the coding region and homeodomain (Fig. 5d). The hallmark Hox14 WFQNQR motif is preserved, however, as is the split homeodomain junction ${ }^{72}$.

Initially, hox 14 genes were thought not to be subject to ancestral regulatory mechanisms that control hox1-hox 13 (ref. ${ }^{71}$ ). However, paddlefish fin buds express hoxd14 posteriorly, consistent with spatially collinear Hox regulation ${ }^{73}$. The bowfin now supports temporal collinearity of hoxd14 expression (see below), and expression in its tailbud reveals another domain exhibiting global collinear regulation. The bowfin hoxd14 pseudogene is still transcribed at high levels, spliced and expressed in highly specific, ancestral patterns. This discovery raises the possibility that, even though bowfin hoxd14 likely no longer codes for a functional protein, it may have some function in the regulation of hox expression and fin patterning and/or other to-be-identified processes. 
a
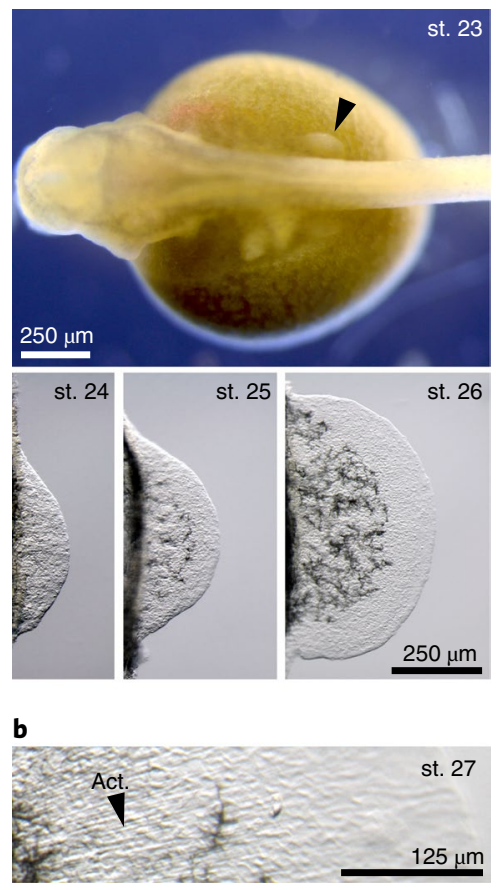

c

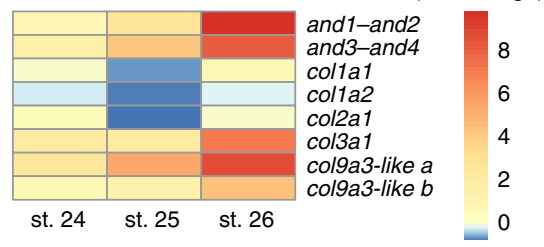

d

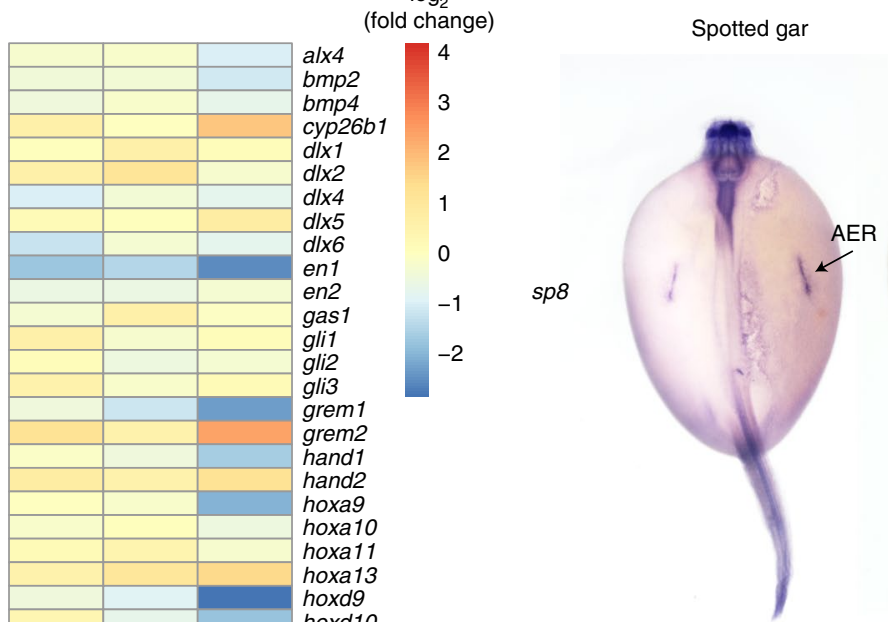

Bowfin

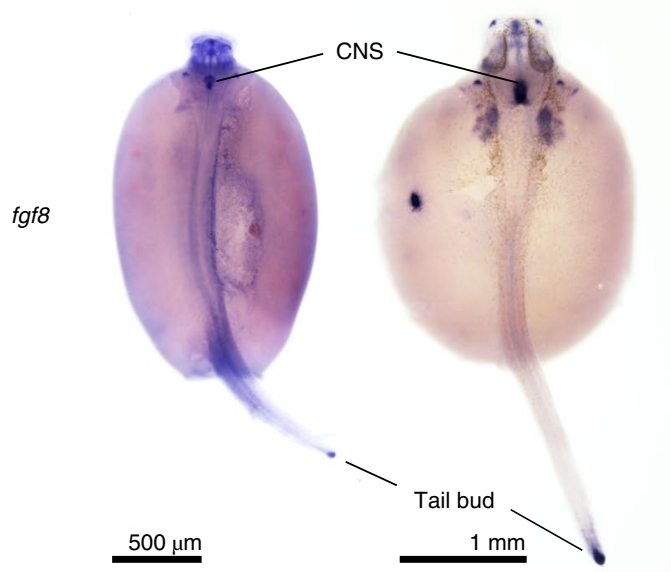

Fig. 6 | Gene expression analyses of developing pectoral fins in bowfin. a, Bowfin developmental stages ${ }^{53}$ used for RNA-seq transcriptome analysis. The fin bud is first visible at stage 23 (black arrowhead, anterior to left). Isolated fin buds showing increasing size from stages 24 to 25 to 26 (anterior to top, distal to right). b. Actinotrichia (Act.) fibrils first appear at stage 27. Pictures in $\mathbf{a}, \mathbf{b}$ are representative of $n=5$ individuals per stage. c, Transcriptional log 2 (fold change) in fin fold structural genes relative to that at stage 23 . Between stages 23 and 26, and1-and2 and and3-and4 show $862-$ and 285 -fold increases, respectively; col3a1, col9a3-like $a$ and col9a3-like $b$ exhibit 133-, 417- and 19-fold increases. Stable expression across stages is observed for col1a1, col1a2 and col2a1, each showing a fold-change range between 0.5 and 1.6. d, Transcriptional $\log _{2}$ (fold change) in expression of fin or limb patterning genes relative to that of stage 23 fin buds. Indicative of their temporal collinearity, expression of hoxa9, hoxa10, hoxd9 and hoxd10 peaks at stage 23 , while expression of the more posterior hoxa11, hoxa13, hoxd11, hoxd12 and hoxd13 peaks at stage 25. Expression of hoxa13 is highest at stage 26 . The highest expression level of hoxd14 is at stages 25 and 26. e, The transcription factor gene sp8 is expressed in the AER of gar (stage 26) and bowfin (stage 23), while fgf8 expression is not detected in the AER of either holostean ( $n=10$ samples per gene and species). CNS, central nervous system.

Unexpected gene expression dynamics during holostean pectoral fin development. Given their predominance among neopterygians, teleosts have long been used as models for paired fin development and as outgroups for tetrapod limb development. However, the teleost pectoral fin endoskeleton has undergone severe reduction since the ancestral bony vertebrate, having lost the metapterygium and proximal-distal long bone articulation. Bowfin is the closest living teleost relative that retains the metapterygium and proximal-distal elaboration of the fin endoskeleton (Extended Data Fig. 5a,b) and thus serves as an essential node connecting appendicular skeletal diversity of ray-finned fishes and those of lobe-finned vertebrates, including tetrapods.

We performed RNA-seq across four early patterning stages of bowfin pectoral fin bud development (Figs. 1b and 6a, Extended Data Fig. 5c, Supplementary Table 25 and Supplementary Note 11), revealing dynamic expression of many known fin or limb patterning genes. The most dramatic expression change in bowfin was in the transcription of structural genes of actinodin and collagen encoding components of the actinotrichia, elastinoid fibrils supporting the early fin fold ${ }^{74}$ (Fig. 6b). The expression of actinodin genes spikes at stage 26, as does that of type III and type IX collagen components (Fig. 6c). By contrast, colla1, colla2 and col2a1 exhibit relatively stable expression across the bowfin stages examined (Fig. 6c) but exhibit dynamic expression and comprise the main structural component of actinotrichia during zebrafish fin development ${ }^{75}$. Genes encoding type III collagens, absent from teleosts genomes $^{75}$, have not been previously implicated in actinotrichia development and may underlie differences between bowfin and teleost fin morphology.

Given the distinct variation in patterning of the endochondral component between holosteans and teleosts, we focused on changes observed in early patterning genes during bowfin development. Temporal and spatial collinearity of Hox cluster gene expression plays critical roles in the patterning of fin and limb skeletons. 
We found collinearity in bowfin hox gene expression levels across early fin bud outgrowth. Peak expression of posterior hox genes tracked with their position within the Hox cluster, including that of hoxd14 with highest expression levels at stages 25 and 26 (Fig. 6d). Other gene expression patterns were consistent with mechanisms described in limbs. Termination of tetrapod limb bud outgrowth is caused by the transcription factor Twist2, which negatively regulates grem 1 expression ${ }^{76}$. Similarly, bowfin twist 2 expression peaked at stage 26, while that of grem 1 peaked at stage 23 and was then downregulated approximately threefold by stage 26 (Fig. 6d). Other correlated expression profiles in bowfin are consistent with known genetic interactions, such as negative regulation of meis genes by cytochrome Cyp26b1 (ref. ${ }^{77}$ ) (Fig. 6d).

Surprisingly, we noticed a lack of expression of the critical limb-development signaling ligand gene $f g f 8$ from the bowfin pectoral fin transcriptome at all stages. By contrast, other fin or limb apical ectodermal ridge (AER) markers are highly expressed across all stages, such as $s p 8$ (Extended Data Fig. $5 \mathrm{~d}$ ), which encodes a transcription factor that directly positively regulates $f g f 8$ expression in the $\mathrm{AER}^{78}$. Using RNA in situ hybridization, we found expected $s p 8$ and $f g f 8$ expression in the central nervous system and tailbud in both bowfin and gar embryos (Fig. 6e). In both holosteans, $s p 8$ was highly expressed in the AER throughout early fin bud development, while fgf8 expression was not detected in the AER (Fig. 6e). These findings are surprising, as $f g f 8$ is expressed in every fin and limb bud in which it has been assessed across gnathostome lineages (for example, refs. ${ }^{79-82}$ ) and is critical for the outgrowth and patterning of these appendages. Even in species that lack a morphological AER, $f g f 8$ is still expressed (for example, refs. ${ }^{83,84}$ ). Thus, the lack of $f g f 8$ expression in the holostean fin bud is entirely unexpected. Given that $s p 8$ was expressed in the AER at high levels (Extended Data Fig. 5d), we hypothesize that changes have occurred in the regulation of $f g f 8$ that remove Sp8 responsiveness in the fin bud, a unique holostean feature. Genomic comparisons (Supplementary Note 11) showed that holosteans possess a complex set of known $f g f 8$ enhancers ${ }^{82}$, some of which are marked as ncOCRs by ATAC-seq (Extended Data Fig. 5e and Supplementary Table 26). We did not detect an obvious enhancer near the $f g f 8$ locus not used or deleted in the bowfin (Extended Data Fig. 5e). As epistasis in regulation of $f g f 8$ expression is likely to be complex, and Fgf8 signaling at large still likely plays critical roles in development apart from the pectoral fin (Fig. 6e), shared enhancer use may be common. Our results reveal an unexpected drift in the genetic control of holostean fin development, potentially to some other fibroblast growth factor (Fgf) signaling genes, and represent a unique case of robustness of appendage development in the absence of early $f g f 8$ regulation (Extended Data Fig. 5d and Supplementary Note 11). As these changes are defining for holosteans and not observed in other fishes or tetrapods, these findings demonstrate the importance of holostean genomic information that challenges long-standing assumptions of core developmental mechanisms and informs new models of appendage patterning.

\section{Discussion}

Bowfin's phylogenetic position and unique suite of ancestral and derived phenotypes make it an important component for understanding vertebrate evolution ${ }^{1,20}$. Our analyses using a chromosome-level genome assembly for this 'living fossil' (ref. ${ }^{20}$ ) show that, despite its derived karyotype with superficially convergent similarities to those of teleosts, its chromosomal organization nevertheless indicates a closer phylogenetic relationship to gar in the holostean clade. The slow-evolving, ancestrally unduplicated genome and the developmental epigenome of the bowfin will be a critical resource for comparative vertebrate genomics and evolutionary developmental biology. After genome sequencing, many challenges remain in determining and functionally assaying non-coding gene-regulatory loci in both model and non-model species. Here, we have identified putative enhancers for this non-model species and illustrate that our ATAC-seq dataset can be easily connected to orthologous enhancers in fish and more distant vertebrate lineages. The bowfin not only informs genome evolution in holostean fishes but also offers valuable insights into the genomic basis of its archetypical anatomy and the many developmental and physiological phenotypes in ray-finned fishes. While this species represents a once-large taxonomic group that is now mostly extinct and thus lost for studying genomic diversity, the bowfin genome adds a major sequenced branch for genetic and developmental exploration to the fish tree of life.

Bowfin and gars have persisted for millions of years. Fortunately, the negative perception of holosteans as 'trash fishes' is changing to include greater appreciation for their evolutionary, ecological and cultural importance and, as shown here, their relevance for understanding vertebrate genome biology. As the Amia genus may not be monotypic ${ }^{2}$, our genome assembly will be of utmost importance to evaluate bowfin's species status and conservation.

\section{Online content}

Any methods, additional references, Nature Research reporting summaries, source data, extended data, supplementary information, acknowledgements, peer review information; details of author contributions and competing interests; and statements of data and code availability are available at https://doi.org/10.1038/ s41588-021-00914-y.

Received: 13 October 2020; Accepted: 13 July 2021; Published online: 30 August 2021

\section{References}

1. Jarvik, J. Basic Structure and Evolution of Vertebrates Vol. 1 (Academic Press, 1980).

2. Burr, B. M. \& Bennett, M. G. in Freshwater Fishes of North America 1 (eds Warren, M. L. \& Burr, M. G.) (John Hopkins University Press, 2014).

Nelson, J. S. Fishes of the World 4th edn (John Wiley, 2006).

4. Near, T. J. et al. Resolution of ray-finned fish phylogeny and timing of diversification. Proc. Natl Acad. Sci. USA 109, 13698-13703 (2012).

5. Betancur, R. R. et al. The tree of life and a new classification of bony fishes. PLoS Curr. 5, ecurrents.tol.53ba26640df0ccaee75bb165c8c26288 (2013).

6. Faircloth, B. C., Sorenson, L., Santini, F. \& Alfaro, M. E. A phylogenomic perspective on the radiation of ray-finned fishes based upon targeted sequencing of ultraconserved elements (UCEs). PLoS ONE 8, e65923 (2013).

7. Braasch, I. et al. The spotted gar genome illuminates vertebrate evolution and facilitates human-teleost comparisons. Nat. Genet. 48 , 427-437 (2016).

8. Hughes, L. C. et al. Comprehensive phylogeny of ray-finned fishes (Actinopterygii) based on transcriptomic and genomic data. Proc. Natl Acad. Sci. USA 115, 6249-6254 (2018).

9. Clarke, J. T., Lloyd, G. T. \& Friedman, M. Little evidence for enhanced phenotypic evolution in early teleosts relative to their living fossil sister group. Proc. Natl Acad. Sci. USA 113, 11531-11536 (2016).

10. Braasch, I. et al. A new model army: emerging fish models to study the genomics of vertebrate Evo-Devo. J. Exp. Zool. B Mol. Dev. Evol. 324, 316-341 (2015).

11. $\mathrm{Du}, \mathrm{K}$. et al. The sterlet sturgeon genome sequence and the mechanisms of segmental rediploidization. Nat. Ecol. Evol. 4, 841-852 (2020).

12. $\mathrm{Bi}, \mathrm{X}$. et al. Tracing the genetic footprints of vertebrate landing in non-teleost ray-finned fishes. Cell 184, 1377-1391 (2021).

13. Cheng, P. et al. The American paddlefish genome provides novel insights into chromosomal evolution and bone mineralization in early vertebrates. Mol. Biol. Evol. 38, 1595-1607 (2020).

14. Braasch, I. \& Postlethwait, J. H. in Polyploidy and Genome Evolution (eds Soltis, P. S. \& Soltis, D. E.) Ch. 17, 341-383 (Springer, 2012).

15. Ravi, V. \& Venkatesh, B. The divergent genomes of teleosts. Annu. Rev. Anim. Biosci. 6, 47-68 (2018).

16. Takezaki, N. Global rate variation in bony vertebrates. Genome Biol. Evol. 10, 1803-1815 (2018).

17. Patterson, C. in Interrelationships of Fishes Vol. Supplement 1 (eds Greenwood, P. H., Miles, R. S. \& Patterson, C.) 233-305 (Academic Press, 1973).

18. Grande, L. An Empirical Synthetic Pattern Study of Gars (Lepisosteiformes) and Closely Related Species, Based Mostly on Skeletal Anatomy. The Resurrection of Holostei 1-863 (American Society of Ichthyologists and Herpetologists, 2010). 
19. Sallan, L. C. Major issues in the origins of ray-finned fish (Actinopterygii) biodiversity. Biol. Rev. Camb. Philos. Soc. 89, 950-971 (2014).

20. Grande, L. \& Bemis, W. E. A comprehensive phylogenetic study of amiid fishes (Amiidae) based on comparative skeletal anatomy. An empirical search for interconnected patterns of natural history. J. Vertebr. Paleontol. 18, 1-696 (1998)

21. Majtanova, Z., Symonova, R., Arias-Rodriguez, L., Sallan, L. \& Rab, P. "Holostei versus Halecostomi" problem: insight from cytogenetics of ancient nonteleost actinopterygian fish, bowfin Amia calva. J. Exp. Zool. B Mol. Dev. Evol. 328, 620-628 (2017).

22. Litman, G. W., Frommel, D., Finstad, J. \& Good, R. A. The evolution of the immune reponse. IX. Immunoglobulins of the bowfin: purification and characterization. J. Immunol. 106, 747-754 (1971).

23. Sire, J. Y., Donoghue, P. C. \& Vickaryous, M. K. Origin and evolution of the integumentary skeleton in non-tetrapod vertebrates. J. Anat. 214, 409-440 (2009).

24. Funk, E., Lencer, E. \& McCune, A. Dorsoventral inversion of the air-filled organ (lungs, gas bladder) in vertebrates: RNAsequencing of laser capture microdissected embryonic tissue. J. Exp. Zool. B Mol. Dev. Evol. 334, 325-338 (2020).

25. Funk, E. C., Breen, C., Sanketi, B. D., Kurpios, N. \& McCune, A. Changes in Nkx2.1, Sox2, Bmp4 and Bmp16 expression underlying the lung-to-gas bladder evolutionary transition in ray-finned fishes. Evol. Dev. 22, 384-402 (2020).

26. Chapman, J. A. et al. Meraculous: de novo genome assembly with short paired-end reads. PLoS ONE 6, e23501 (2011).

27. Putnam, N. H. et al. Chromosome-scale shotgun assembly using an in vitro method for long-range linkage. Genome Res. 26, 342-350 (2016).

28. Lieberman-Aiden, E. et al. Comprehensive mapping of long-range interactions reveals folding principles of the human genome. Science $\mathbf{3 2 6}$ 289-293 (2009).

29. Ohno, S. et al. Microchromosomes in holocephalian, chondrostean and holostean fishes. Chromosoma 26, 35-40 (1969).

30. Pasquier, J. et al. Gene evolution and gene expression after whole genome duplication in fish: the PhyloFish database. BMC Genomics 17, 368 (2016)

31. Holt, C. \& Yandell, M. MAKER2: an annotation pipeline and genome-database management tool for second-generation genome projects. BMC Bioinformatics 12, 491 (2011).

32. Emms, D. M. \& Kelly, S. OrthoFinder: phylogenetic orthology inference for comparative genomics. Genome Biol. 20, 238 (2019).

33. Feron, R. et al. RADSex: a computational workflow to study sex determination using restriction site-associated DNA sequencing data. Mol. Ecol. Resour. 21, 1715-1731 (2021).

34. Sacerdot, C., Louis, A., Bon, C., Berthelot, C. \& Roest Crollius, H. Chromosome evolution at the origin of the ancestral vertebrate genome. Genome Biol. 19, 166 (2018).

35. Simakov, O. et al. Deeply conserved synteny resolves early events in vertebrate evolution. Nat. Ecol. Evol. 4, 820-830 (2020).

36. Moret, B. M. E., Tang, J., Wang, L.-S. \& Warnow, T. Steps toward accurate reconstructions of phylogenies from gene-order data. J. Comput. Syst. Sci. 65, 508-525 (2002).

37. Lin, Y., Hu, F., Tang, J. \& Moret, B. M. Maximum likelihood phylogenetic reconstruction from high-resolution whole-genome data and a tree of 68 eukaryotes. Pac. Symp. Biocomput. 2013, 285-296 (2013).

38. Emms, D. M. \& Kelly, S. STAG: species tree inference from all genes. Preprint at bioRxiv https://doi.org/10.1101/267914 (2018).

39. Wcisel, D. J., Ota, T., Litman, G. W. \& Yoder, J. A. Spotted gar and the evolution of innate immune receptors. J. Exp. Zool. B Mol. Dev. Evol. 328, 666-684 (2017).

40. Trowsdale, J. The MHC, disease and selection. Immunol. Lett. 137 1-8 (2011).

41. Ohta, Y. et al. Primitive synteny of vertebrate major histocompatibility complex class I and class II genes. Proc. Natl Acad. Sci. USA 97, 4712-4717 (2000).

42. Grimholt, U. MHC and evolution in teleosts. Biology 5, 6 (2016).

43. Flajnik, M. F. A cold-blooded view of adaptive immunity. Nat. Rev. Immunol. 18, 438-453 (2018).

44. Fillatreau, S. et al. The astonishing diversity of Ig classes and B cell repertoires in teleost fish. Front. Immunol. 4, 28 (2013).

45. Mirete-Bachiller, S., Olivieri, D. N. \& Gambon-Deza, F. Immunoglobulin T genes in Actinopterygii. Fish Shellfish Immunol. 108, 86-93 (2021).

46. Aderem, A. \& Ulevitch, R. J. Toll-like receptors in the induction of the innate immune response. Nature 406, 782-787 (2000).

47. Fitzgerald, K. A. \& Kagan, J. C. Toll-like receptors and the control of immunity. Cell 180, 1044-1066 (2020).

48. Aoki, T., Hikima, J., Hwang, S. D. \& Jung, T. S. Innate immunity of finfish: primordial conservation and function of viral RNA sensors in teleosts. Fish Shellfish Immunol. 35, 1689-1702 (2013).
49. Kawasaki, K. et al. SCPP genes and their relatives in gar: rapid expansion of mineralization genes in Osteichthyans. J. Exp. Zool. B Mol. Dev. Evol. 328, 645-665 (2017).

50. Qu, Q., Haitina, T., Zhu, M. \& Ahlberg, P. E. New genomic and fossil data illuminate the origin of enamel. Nature 526, 108-111 (2015).

51. Kawasaki, K. et al. Coevolution of enamel, ganoin, enameloid, and their matrix SCPP genes in osteichthyans. iScience 24, 102023 (2021)

52. Buenrostro, J. D., Giresi, P. G., Zaba, L. C., Chang, H. Y. \& Greenleaf, W. J. Transposition of native chromatin for fast and sensitive epigenomic profiling of open chromatin, DNA-binding proteins and nucleosome position. Nat. Methods 10, 1213-1218 (2013).

53. Ballard, W. W. Stages and rates of normal development in the holostean fish, Amia calva. J. Exp. Zool. 238, 337-354 (1986).

54. Heinz, S. et al. Simple combinations of lineage-determining transcription factors prime cis-regulatory elements required for macrophage and B cell identities. Mol. Cell 38, 576-589 (2010).

55. Armstrong, J. et al. Progressive Cactus is a multiple-genome aligner for the thousand-genome era. Nature 587, 246-251 (2020).

56. Visel, A., Minovitsky, S., Dubchak, I. \& Pennacchio, L. A. VISTA Enhancer Browser-a database of tissue-specific human enhancers. Nucleic Acids Res. 35, D88-D92 (2007)

57. Yuan, X. et al. Heart enhancers with deeply conserved regulatory activity are established early in zebrafish development. Nat. Commun. 9, 4977 (2018).

58. Abbasi, A. A. et al. Human intronic enhancers control distinct sub-domains of Gli3 expression during mouse CNS and limb development. BMC Dev. Biol. 10, 44 (2010).

59. Adachi, N., Robinson, M., Goolsbee, A. \& Shubin, N. H. Regulatory evolution of Tbx5 and the origin of paired appendages. Proc. Natl Acad. Sci. USA 113, 10115-10120 (2016).

60. Menke, D. B., Guenther, C. \& Kingsley, D. M. Dual hindlimb control elements in the Tbx4 gene and region-specific control of bone size in vertebrate limbs. Development 135, 2543-2553 (2008).

61. Zhang, W. et al. Spatial-temporal targeting of lung-specific mesenchyme by a Tbx4 enhancer. BMC Biol. 11, 111 (2013)

62. Vernimmen, D. Uncovering enhancer functions using the $\alpha$-globin locus. PLoS Genet. 10, e1004668 (2014).

63. Huang, P. et al. Comparative analysis of three-dimensional chromosomal architecture identifies a novel fetal hemoglobin regulatory element. Genes Dev. 31, 1704-1713 (2017).

64. Ulianov, S. V. et al. Activation of the $\alpha$-globin gene expression correlates with dramatic upregulation of nearby non-globin genes and changes in local and large-scale chromatin spatial structure. Epigenetics Chromatin 10, 35 (2017).

65. Pijuan-Sala, B. et al. Single-cell chromatin accessibility maps reveal regulatory programs driving early mouse organogenesis. Nat. Cell Biol. 22, 487-497 (2020).

66. Tena, J. J. et al. Comparative epigenomics in distantly related teleost species identifies conserved cis-regulatory nodes active during the vertebrate phylotypic period. Genome Res. 24, 1075-1085 (2014).

67. Li, Y. et al. Dynamic transcriptional and chromatin accessibility landscape of medaka embryogenesis. Genome Res. 30, 924-937 (2020).

68. Graham, J. B. Air-Breathing Fishes (Academic Press, 1997).

69. Meyer, A. et al. Giant lungfish genome elucidates the conquest of land by vertebrates. Nature 590, 284-289 (2021).

70. Wang, K. et al. African lungfish genome sheds light on the vertebrate water-to-land transition. Cell 184, 1362-1376 (2021).

71. Kuraku, S. et al. Noncanonical role of Hox 14 revealed by its expression patterns in lamprey and shark. Proc. Natl Acad. Sci. USA 105, 6679-6683 (2008)

72. Powers, T. P. \& Amemiya, C. T. Evidence for a Hox14 paralog group in vertebrates. Curr. Biol. 14, R183-R184 (2004).

73. Tulenko, F. J. et al. HoxD expression in the fin-fold compartment of basal gnathostomes and implications for paired appendage evolution. Sci. Rep. 6, 22720 (2016).

74. Zhang, J. et al. Loss of fish actinotrichia proteins and the fin-to-limb transition. Nature 466, 234-237 (2010).

75. Duran, I. et al. Collagen duplicate genes of bone and cartilage participate during regeneration of zebrafish fin skeleton. Gene Expr. Patterns 19, 60-69 (2015).

76. Wade, C., Brinas, I., Welfare, M., Wicking, C. \& Farlie, P. G. Twist2 contributes to termination of limb bud outgrowth and patterning through direct regulation of Grem1. Dev. Biol. 370, 145-153 (2012).

77. Yashiro, K. et al. Regulation of retinoic acid distribution is required for proximodistal patterning and outgrowth of the developing mouse limb. Dev. Cell 6, 411-422 (2004)

78. Kawakami, Y. et al. Sp8 and Sp9, two closely related buttonhead-like transcription factors, regulate $\mathrm{Fgf8}$ expression and limb outgrowth in vertebrate embryos. Development 131, 4763-4774 (2004). 
79. Gillis, J. A., Dahn, R. D. \& Shubin, N. H. Shared developmental mechanisms pattern the vertebrate gill arch and paired fin skeletons. Proc. Natl Acad. Sci. USA 106, 5720-5724 (2009).

80. Tulenko, F. J. et al. Fin-fold development in paddlefish and catshark and implications for the evolution of the autopod. Proc. Biol. Sci. 284, 20162780 (2017)

81. Hodgkinson, V. S., Ericsson, R., Johanson, Z. \& Joss, J. M. P. The apical ectodermal ridge in the pectoral fin of the Australian lungfish (Neoceratodus forsteri): keeping the fin to limb transition in the fold. Acta Zool. 90, 253-263 (2009).

82. Gehrke, A. R. \& Shubin, N. H. Cis-regulatory programs in the development and evolution of vertebrate paired appendages. Semin. Cell Dev. Biol. 57, 31-39 (2016).

83. Doroba, C. K. \& Sears, K. E. The divergent development of the apical ectodermal ridge in the marsupial Monodelphis domestica. Anat. Rec. 293, 1325-1332 (2010)

84. Purushothaman, S., Elewa, A. \& Seifert, A. W. Fgf-signaling is compartmentalized within the mesenchyme and controls proliferation during salamander limb development. eLife 8, e48507 (2019).

85. Negrisolo, E. et al. Different phylogenomic approaches to resolve the evolutionary relationships among model fish species. Mol. Biol. Evol. 27, 2757-2774 (2010)
86. Nikaido, M. et al. Coelacanth genomes reveal signatures for evolutionary transition from water to land. Genome Res. 23, 1740-1748 (2013).

87. Frazer, K. A., Pachter, L., Poliakov, A., Rubin, E. M. \& Dubchak, I. VISTA: computational tools for comparative genomics. Nucleic Acids Res. 32 W273-W279 (2004).

88. Brudno, M. et al. Glocal alignment: finding rearrangements during alignment. Bioinformatics 19, i54-i62 (2003).

Publisher's note Springer Nature remains neutral with regard to jurisdictional claims in published maps and institutional affiliations.

(c) (i) Open Access This article is licensed under a Creative Commons Attribution 4.0 International License, which permits use, sharing, adaptation, distribution and reproduction in any medium or format, as long as you give appropriate credit to the original author(s) and the source, provide a link to the Creative Commons license, and indicate if changes were made. The images or other third party material in this article are included in the article's Creative Commons license, unless indicated otherwise in a credit line to the material. If material is not included in the article's Creative Commons license and your intended use is not permitted by statutory regulation or exceeds the permitted use, you will need to obtain permission directly from the copyright holder. To view a copy of this license, visit http://creativecommons.org/licenses/by/4.0/.

(c) The Author(s) 2021 


\section{Methods}

Animal work. Bowfin work was performed in compliance with ethical guidelines and approved under Institutional Animal Care and Use Committee protocols from Nicholls State University (IA046/IA053), Michigan State University (10/16-179-00) and Cornell University (2006-0013). Bowfins for genome sequencing, sex-determination analysis and immune transcriptomics were sampled from the Louisiana (USA) population as detailed below. Bowfin embryos and larvae (unsexed) for developmental and fin bud transcriptomics, ATAC-seq and RNA in situ hybridization were collected from nests of the Oneida Lake (New York, USA) population and then raised in the laboratory as previously described ${ }^{25}$ (Supplementary Note 11) until sampling at the desired developmental stages ${ }^{53}$. Spotted gar embryos (unsexed) were obtained from the Louisiana population, raised and fixed as previously described ${ }^{89}$, and protocols were approved by the Nicholls State University (IA053) and the Michigan State University (10/16-179-00) institutional animal care and use committees.

Genome sequencing and assembly. DNA was extracted from blood of a single, adult, wild male (phenotypic sex was confirmed by gonadal observation) collected from the Atchafalaya River Basin in Louisiana, USA (Supplementary Note 1). A de novo assembly was constructed using a paired-end sequencing library (mean assembly-based insert size, $\sim 410 \mathrm{bp}$ ) with Meraculous version 2.2.2.5 $\left(\right.$ ref. ${ }^{26}$ ) ( $k$-mer size 55 ; minimum $k$-mer frequency 55 ; diploid nonredundant haplotigs). Input data consisted of 433.5 million read pairs sequenced from the paired-end library, totaling $122 \mathrm{~Gb}$ after trimming for quality, sequencing adaptors and mate pair adaptors using Trimmomatic version 0.38 (ref. ${ }^{90}$ ) (parameters: 'PE ILLUMINACLIP LEADING:20 TRAILING:20 SLIDINGWINDOW:13:20 MINLEN:23')

A Chicago library was prepared as previously described ${ }^{27}$. Briefly, $\sim 500 \mathrm{ng}$ HMW gDNA (mean fragment length $50 \mathrm{~kb}$ ) was reconstituted into chromatin in vitro and fixed in $1 \%$ formaldehyde for $15 \mathrm{~min}$ at room temperature. Fixed chromatin was digested with DpnII, $5^{\prime}$ overhangs were filled in with biotinylated nucleotides, and free blunt ends were ligated. After ligation, cross-links were reversed, and DNA was purified from protein. Purified DNA was treated to remove biotin that was not internal to ligated fragments. DNA was then sheared to a mean fragment size of $\sim 350 \mathrm{bp}$, and sequencing libraries were generated using NEBNext Ultra enzymes and Illumina-compatible adaptors. Biotin-containing fragments were isolated using streptavidin beads before PCR enrichment of each library. Libraries were sequenced on an Illumina HiSeq platform to produce 177 million $2 \times 101$-bp paired-end reads, which provided $89.9 \times$ physical coverage of the $k$-mer-based estimated genome size of $0.91 \mathrm{~Gb}$ (1-50-kb pairs).

A Hi-C library was prepared as previously described ${ }^{28}$. For each library, chromatin was fixed in place in the nucleus in $1 \%$ formaldehyde for $10 \mathrm{~min}$ at room temperature and then extracted. Fixed chromatin was digested with DpnII, 5 ' overhangs were filled in with biotinylated nucleotides, and then free blunt ends were ligated. After ligation, cross-links were reversed, and DNA was purified from protein. Purified DNA was treated to remove biotin that was not internal to ligated fragments. DNA was sheared to a mean fragment size of $\sim 350 \mathrm{bp}$, and sequencing libraries were generated using NEBNext Ultra enzymes and Illumina-compatible adaptors. Biotin-containing fragments were isolated using streptavidin beads before PCR enrichment of each library. Libraries were sequenced on an Illumina HiSeq platform to produce 527 million $2 \times 151$-bp paired-end reads, which provided $22,887 \times$ physical coverage of the $k$-mer-based estimated genome size (1-50-kb pairs).

Input de novo assembly, shotgun, Chicago library and Hi-C library reads were used as input data for HiRise ${ }^{27}$ to scaffold genome assemblies with an iterative analysis. First, shotgun and Chicago library sequences were aligned to the draft input assembly using a modified SNAP read mapper (http://snap.cs.berkeley.edu). Separations of Chicago read pairs mapped within draft scaffolds were analyzed by HiRise to produce a likelihood model for genomic distance between read pairs. The model was used to identify and break putative misjoins, to score prospective joins and to make joins above a likelihood threshold. After aligning and scaffolding Chicago data, Dovetail Hi-C library sequences were aligned and scaffolded following the same method. After scaffolding, shotgun sequences were used to close gaps between contigs to generate the final genome assembly (AmiCal1).

Hi-C contact mapping. Raw Hi-C reads were trimmed with Trimmomatic version 0.38 (ref. ${ }^{90}$ ) (parameters: 'PE, ILLUMINACLIP:adapter_contamination_ sequences.txt:1:30:7 MINLEN:25'). BWA version 0.7.17 (ref. ${ }^{91}$ ) was used to map forward and reverse reads to the final genome assembly independently with the following parameters: 'bwa mem -A1 -B4 -E50 -L0'. Downstream analyses were performed with the HiCExplorer version 3.6 toolkit $^{92}$. A Hi-C matrix was constructed from mapped reads at $10-\mathrm{kb}$ resolution with the hicBuildMatrix tool. The hicMergeMatrixBins tool was used to further bin the matrix into $50-\mathrm{kb}, 100-\mathrm{kb}, 200-\mathrm{kb}, 300-\mathrm{kb}, 400-\mathrm{kb}$ and $500-\mathrm{kb}$ bin size matrices. All matrices were corrected with hicCorrectMatrix with the filter threshold parameter set to ' -1.55 '. TADs were called with hicFindTADs using the following parameters: '-correctForMultipleTesting fdr-thresholdComparisons 0.05-delta 0.01'. Hi-C contact maps were plotted using the hicPlotMatrix tool and pyGenomeTracks version 3.6 (ref. ${ }^{93}$ )
Repeat analysis. A custom bowfin repeat database was constructed using Repeat Modeler version 1.0.8 (ref. ${ }^{94}$ ) with default parameters and combined with custom repeat libraries used for gar $^{7}$ and all repeats from the Vertebrata Repbase $^{95}$ (downloaded 15 November 2017). These repeat elements were input to RepeatMasker ${ }^{96}$ within MAKER version 2.31 (ref. ${ }^{31}$ ) during gene annotation. Repeat proteins were identified by searching against a library packaged with MAKER via RepeatRunner ${ }^{97}$. The new combined repeat library was used to characterize repeats in both bowfin and spotted gar. RepeatMasker functions 'calcDivergenceFromAlign.pl' and 'createRepeatLandscape.pl' (ref. ${ }^{96}$ ) generated repeat landscapes for both species.

Gene annotation. Protein-coding genes were annotated with a bowfin reference transcriptome from multiple adult tissues ${ }^{7,30}$ as evidence. The EST2genome function in MAKER version 2.31 (ref. ${ }^{31}$ ) was used identify putative bowfin genes based on BLAST ${ }^{98}$ and Exonerate ${ }^{99}$ transcript alignments. Best-scoring genes with an annotation edit distance of 0.2 or less were used to train hidden Markov models with SNAP ${ }^{100}$ and AUGUSTUS ${ }^{101}$ as previously described ${ }^{102}$. Ensembl and RefSeq protein sequences from other vertebrate species were used as additional evidence: gar (LepOcu1), coelacanth (LatCha1), mouse (GRCm38.p5), chicken (Gallus_gallus-5.0), human (GRCh38.p10), Xenopus (JGI 4.2), anole lizard (AnoCar2.0), zebrafish (GRCz10), medaka (HdrR), arowana (GCA_001624265.1, ASM162426v1) and elephant shark (GCA_000165045.2, Callorhinchus_milii6.1.3). Proteins were aligned to the genome using BLAST and Exonerate with default options, guiding gene predictions by HMMs (from SNAP and AUGUSTUS) in the MAKER workflow. MAKER output all predicted genes with and without transcript and protein evidence (MAKER-Max). Pfam protein domains ${ }^{103}$ were identified within the MAKER-Max gene set using HMMER version 3.0b3 (hmmrscan $E$ value $\left.<1 \times 10^{-5}\right)^{104}$. MAKER-Max genes with transcript or protein or Pfam domain support were retained as the final MAKER-Standard gene set ${ }^{105}$.

We ran OrthoFinder version 1.1.3 (ref. ${ }^{32}$ ) (fastME distance method parameters: '-t 20 -M msa -A mafft -T FastTree') to identify orthologous genes between bowfin and other vertebrates using protein sequences of the longest isoforms from the same species set used for MAKER annotation.

Bowfin sex-determination analyses. Reference genome-based and genome-free Pool-seq approaches to compare 30 adult bowfin males with 30 adult females (phenotypic sex was confirmed by gonadal observation) from the Louisiana population are described in Supplementary Note 3.

\section{Genome structure and gene-order analyses. Orthologous genes were extracted} from reconciled gene trees built with the Ensembl Compara pipeline ${ }^{106}$. The reconciled gene trees contain 78 species, including 55 fish, 18 other vertebrates and five non-vertebrate outgroups. Briefly, starting with the set of predicted coding sequences, we performed an all-against-all $\mathrm{BLAST}^{98}$, followed by clustering with hcluster_sg ${ }^{107}$ to define gene families, multiple-alignment inference using $\mathrm{M}-\mathrm{Coffee}^{108}$ and phylogenetic tree construction with TreeBeST ${ }^{106}$. From these trees, gene families were defined as groups of genes that are derived from the same ancestral gene. Depending on the set of species used, we chose different ancestral species to define the families, each time taking the most recent common ancestor (see below).

We identified pairs of chromosomes between species sharing significantly more orthologs than expected after random gene shuffling $\left(P<0.05, \chi^{2}\right.$ test; Yates correction for small sample size, Bonferroni correction for multiple testing). Karyotype comparisons with spotted gar were based on all orthologous genes inherited from the Neopterygii ancestor $(n=16,398$ orthologous genes between bowfin and gar; $n=14,374$ orthologous genes between medaka and gar). Comparisons with chicken were based on all orthologous genes inherited from the Euteleostomi ancestor $(n=8,219$ orthologous genes between bowfin and chicken; $n=7,835$ orthologous genes between gar and chicken).

We used a distance-based method to reconstruct the phylogeny of 14 species using gene-order data from high-confidence orthologs inherited from the common Euteleostomi ancestor, reducing all studied genomes to 3,223 marker genes: 1,527 were singletons in all species; 1,614 were present in one copy in bowfin, gar, chicken and Xenopus and one or two copies in post-TGD teleosts; 82 were present in one copy in all non-teleosts and two copies in all teleosts. Intervals between these marker genes cover $>60 \%$ of the most fragmented genomes and $>80 \%$ of all other genomes and are arranged into 16,064 gene adjacencies that exist in at least one study species. Differences in genome assembly qualities and gene duplications resulted in varying numbers of adjacent gene markers, ranging from 3,028 (Amazon molly) to 3,800 (arowana).

Normalized breakpoint distances between pairs of genomes were computed as the number of gene adjacencies that exist in G1 but not in G2, normalized to the number of adjacencies in G1, where G1 is the genome with the smallest number of adjacencies, in line with previously reported adjustments ${ }^{109,110}$. Species trees were reconstructed on the resulting distance matrix using NJ, minimum evolution (FastME) and UPGMA approaches, with bootstrap replicates generated by resampling 100 times with replacement from the 16,064 gene adjacencies. Bootstrapped NJ trees were used to evaluate the $95 \%$ confidence interval of the branch-length difference of bowfin versus gar and bowfin versus teleosts (see 
Supplementary Note 5 for details). To address the possibility that adjacency losses in teleosts result from loss of TGD-duplicated genes instead of rearrangements, we explicitly ignored any adjacency loss for marker genes on alternative TGD-derived regions, which did not affect the results.

Comparing Holostei versus Halecostomi scenarios within a maximum parsimony framework, we treated each of the 566 Neopterygian-specific gene adjacencies as an independent character, either absent or present in each Neopterygian genome, and placed adjacency gains and losses on the two alternative phylogenies according to the assumptions of Dollo's parsimony ${ }^{111}$, as chances are extremely low that distinct rearrangements would (re)create the same gene adjacency. To assess whether one scenario was significantly more parsimonious than the other, we used the $\mathrm{KH}$ test (Phylip package ${ }^{112}$, dollop program).

Gene-family analyses. Immune, SCPP and Hox genes were annotated and analyzed as detailed in Supplementary Notes 6, 7 and 10, respectively. Generally, spotted gar and/or other vertebrate sequences were used in BLAST searches ${ }^{98}$ against the bowfin genome assembly to generate manually curated annotations further supported by diverse bowfin transcriptomic evidence (described below and published $\left.{ }^{7,30}\right)$. Orthologies were assigned based on phylogenetic and synteny evidence as described in the respective Supplementary Notes.

Developmental transcriptomics. Total RNA was extracted (Qiagen RNeasy Min Plus) from RNAlater-preserved (Ambion), unsexed embryos or larvae from the Oneida Lake population at Ballard stages 22-23, 23-24, 24-25, 26-27, 28-29 and $30-31$ (ref. ${ }^{53}$ ). Stage $22-23$ was defined as the bowfin phylotypic stage by similarity to the phylotypic stages of zebrafish and medaka ${ }^{66}$. Five embryos were pooled for stages 22-23 and 24-25; one embryo or larva was used for stages 23-24, 26-27, 28-29 and 30-31. Libraries were generated with the Illumina TruSeq Stranded mRNA Library Preparation Kit with IDT for Illumina Unique Dual Index and sequenced on an Illumina NextSeq version 2.5 (Mid Output flow cell, $2 \times 75$-bp paired-end format, 150 -cycle version 2.5 NextSeq 500 reagent cartridge). After base calling with Illumina Real Time Analysis (RTA) version 2.4.11, sequences were demultiplexed and converted to FastQ format with Illumina bcl2fastq version 2.19.1. Read pairs were trimmed for quality and adaptor sequences with Trimmomatic version 0.38 (ref. ${ }^{90}$ ) using the same parameters as described above for $\mathrm{Hi}-\mathrm{C}$ reads. Remaining paired-end reads were mapped to the repeat-masked bowfin genome with Bowtie 2 (ref. ${ }^{113}$ ) ('very-sensitive' option, other parameters set to default). Reads mapping to the mitochondrial genome were removed with 'removeChrom.py' (https://github.com/harvardinformatics/ATAC-seq/blob/ master/atacseq/removeChrom.py), reads from PCR duplicates were removed with Picard Tools version 2.18.1 ('mark duplicates' function) (https://broadinstitute. github.io/picard/), and SAMtools view ('-b -q 10') was used to remove poorly mapped reads ${ }^{114}$. Reads were visualized in $\mathrm{IGV}^{115}$ after normalization with BPM (bin size, 25) with the deepTools version 3.1.1 (ref. ${ }^{116}$ ) bamCoverage tool.

Immune tissue transcriptomics. Immune tissues (gill, spleen, intestine) were dissected from a single adult female bowfin from Bayou Chevreuil, Louisiana. RNA was extracted from each individual tissue (Qiagen RNeasy kit), quantified (Thermo Fisher NanoDrop and Agilent Bioanalyzer) and pooled in equal amounts before library barcoding. Sequencing reads from the NovaSeq 6000 were quality trimmed using Trimmomatic version 0.38 (ref. ${ }^{90}$ ) and assembled into transcripts using genome-guided Trinity version 2.8 .5 (ref. ${ }^{117}$ ) (Supplementary Note 6).

Fin bud expression analyses. Whole, unsexed bowfin embryos from the Oneida Lake population were incubated overnight in RNAlater at $4^{\circ} \mathrm{C}$, followed by dissection of pectoral fin buds using tungsten needles with three replicates of six to seven animals collected at the desired stages. Tissues were dissolved by incubation in RLT Plus. RNA was isolated from both whole animals (for cDNA cloning) and dissected fin buds using the RNeasy Plus Micro kit (Qiagen). For standard cloning, cDNA libraries were produced from RNA extracted from stage 23 and stage 25 single whole embryos and from stage 26 fin buds using the SuperScript IV Reverse Transcriptase System (Invitrogen). Following polyA selection, stranded mRNA-seq libraries were produced using the PrepX RNA-seq Library Kit for Illumina (IntegenX) from stage 23, 24, 25 and 26 fin bud RNA. For each developmental stage, two replicate libraries were sequenced with 75-bp single-end reads, and a third was sequenced with 150-bp paired-end reads on an Illumina NextSeq machine. Reads were filtered using Trimmomatic version 0.38 (ref. ${ }^{90}$ ), and ribosomal RNA reads were removed using SortMeRNA ${ }^{118}$. Filtered non-rRNA reads were aligned to the bowfin nuclear transcriptome using CLC Genomics Workbench (Qiagen). The transcriptome toolkit in CLC Genomics Workbench was used to calculate RPKM values, fold change and analysis of differential gene expression. Heatmaps to visualize gene expression dynamics were created using the Pretty Heatmaps package in R (https://rdrr.io/cran/pheatmap/).

Assay for transposase-accessible chromatin with sequencing analysis. ATAC-seq library preparation and amplification with barcoding was performed using a modification of published protocols ${ }^{52,119}$. Samples were taken from the same unsexed clutches (Oneida Lake population) and stages as for the developmental transcriptome series, covering stages 21-31 (see above). One whole embryo per stage was dissociated into a cell solution with $0.125 \%$ collagenase (Sigma, C9891) at $37^{\circ} \mathrm{C}$ until completely dissociated and then strained (100- $\mu \mathrm{M}$ filter) and counted on an improved Neubauer chamber. In total, 100,000 cells were collected and lysed per sample for DNA transposition and PCR amplification. Agencourt AMPure XP beads were used to clean up PCR products. ATAC-seq libraries were individually barcoded, pooled and sequenced with Illumina NextSeq version 2 (High Output flow cell, $2 \times 75$-bp paired-end format, 150 -cycle version 2 NextSeq 500 reagent cartridge). Base calling, demultiplexing, FastQ format conversion, read trimming, mapping to the bowfin genome, alignment filtering and data visualization of ATAC-seq reads in IGV ${ }^{115}$ were performed as described above for developmental transcriptome RNA-seq data.

To identify OCRs, ATAC-seq peaks were called with MACS2 version 2.2.6 (ref. ${ }^{120}$ ) (parameters: 'callpeak -f BAMPE -g 767196669 -B -q 0.05 -s 75call-summits'). BEDTools ${ }^{121}$ 'subtract $-A^{\prime}$ ' was used to subtract exon coordinates (UTRs and coding regions) from OCR coordinates in each library to generate ncOCRs, defined as OCRs with no overlap ( $0 \mathrm{bp})$ with any MAKER-annotated exon. BEDTools ${ }^{121}$ merge was used to merge OCRs and ncOCRs within libraries and stages (across replicates) as well as across stages for a global picture of open chromatin through development. OCRs merged within stage were annotated with HOMER $^{54}$ 'annotatePeaks.pl' ('-size 'given' -annStats'). Transcriptional start sites were defined from $-1 \mathrm{~kb}$ to $+100 \mathrm{bp}$; transcriptional termination sites were defined from $-100 \mathrm{bp}$ to $+1 \mathrm{~kb}$. OCR overlap and uniqueness between developmental stages were quantified with HOMER ${ }^{54}$ 'mergePeaks' (parameters: '-d given -venn'). Pairwise Jaccard distances were calculated between stages and replicates with the BEDTools Jaccard option and visualized with a heatmap created with Heatmapper ${ }^{122}$ and a PCA plot created with ggplot2 (ref. ${ }^{123}$ ). Read pileup and merged OCRs from MACS2 were visualized in $\mathrm{IGV}^{115}$ after normalization with BPM (bin size, 25) with the deepTools version 3.1.1 (ref. ${ }^{116}$ ) bamCoverage tool.

\section{Whole-genome alignments and OCR overlap. We used Progressive} Cactus version 1.2.3 (ref. ${ }^{55}$ ) to align bowfin, gar, zebrafish (GRCz11), human (hg38) and mouse (GRCm38) genomes given the phylogeny (((gar, bowfin), zebrafish), (human, mouse)) using default parameters. After completion of alignment, we used the 'halLiftover' tool ${ }^{55}$ to identify homologous elements between species as follows: 'halLiftover-noDupes vertebrates.hal $<$ reference species $>$ reference_sp_coordinates.bed < target species $>$ target_sp_ coordinates.bed'. We used this approach to lift bowfin OCRs to all other species as well as lift mouse OCRs ${ }^{65}$, human and mouse Vista enhancers ${ }^{56}$ and our previously generated gar-centric $\mathrm{CNEs}^{7}$ to the bowfin genome. Gar-centric CNEs (sensu ${ }^{7}$; 156,087 total elements) were defined as $\geq 50$-bp phastCons elements from a 13 -way MultiZ vertebrate WGA, filtered for genic and repeat regions to obtain $\mathrm{CNEs}^{7}$. We counted all $\geq 50$-bp lifted elements as 'conserved' between the reference and target species and summarized overlap between the conserved elements from other species with bowfin OCRs using BEDTools (parameters: 'intersect -wa -f 0.33'). We counted all conserved elements that have at least $33 \%$ of their length overlapping a bowfin OCR. We applied this method to all bowfin OCRs and ncOCRs and separately to those represented in at least two developmental stages (defined by HOMER mergePeaks). Locations of published ray-finned fish UCEs ${ }^{6}$ (366 total elements) in the bowfin genome were established through BLASTN $\left(E\right.$ value $\left.<1 \times 10^{-5}\right)$. Ray-finned fish UCEs (sensu ${ }^{6}$ ) have been defined as ultraconserved, $\geq 120$-bp single-copy nuclear DNA elements with $\geq 80 \%$ sequence identity across a five-species teleost WGA, covering both coding and non-coding sequence space ${ }^{6}$.

Local genomic sequence-conservation analyses. Repeat-masked genomic regions surrounding $t b \times 4$ and $f g f 8$ in bowfin and other vertebrates were aligned with mVISTA $^{87}$ using SLAGAN ${ }^{88}$ as detailed in Supplementary Notes 9 and 11, respectively.

RNA in situ hybridization. For RNA in situ hybridization and skeletal staining, unsexed embryos of bowfin (Oneida Lake population) and gar (Louisiana population) were fixed overnight at $4{ }^{\circ} \mathrm{C}$ in $4 \%$ paraformaldehyde in PBS, washed twice for $5 \mathrm{~min}$ in PBS with $0.1 \%$ Tween, washed once in $100 \%$ methanol for $5 \mathrm{~min}$ and stored in $100 \%$ methanol at $-20^{\circ} \mathrm{C}$. Amplified bowfin and gar cDNA (see Supplementary Note 11 for primer sets) was cloned into pGEM-T Easy (Promega). Antisense probes were synthesized as previously described ${ }^{124}$, and whole-mount in situ hybridization was performed using a standard embryo $\operatorname{protocol}^{125}(n=10$ per gene and species).

Reporting Summary. Further information on research design is available in the Nature Research Reporting Summary linked to this article.

\section{Data availability}

The bowfin reference genome assembly (AmiCal1) is available at GenBank under the accession number PESF00000000; raw reads are available at the Sequence Read Archive under accession numbers SRR14766073-SRR14766075. Transcriptomic and ATAC-seq reads are available under accession numbers SRP281665 and SRP252716; assembled transcripts are available under accession number GIOP00000000. The MAKER gene annotation is available at https:// github.com/AndrewWT/AmiaGenomics. Data for synteny analyses and the 
gene-order phylogeny are available at https://github.com/DyogenIBENS/ BowfinGOPhylogeny. Source data are provided with this paper.

\section{Code availability}

Custom scripts and workflows for the Pool-seq sex-determination analysis are available at https://github.com/RomainFeron/paper-sexdetermination-bowfin and for the gene-order phylogeny at https://github.com/DyogenIBENS/ BowfinGOPhylogeny. All other software was used as described in detail in the Methods and the Nature Research Reporting Summary.

\section{References}

89. Braasch, I. et al. Connectivity of vertebrate genomes: paired-related homeobox (Prrx) genes in spotted gar, basal teleosts, and tetrapods. Comp. Biochem. Physiol. C Toxicol. Pharmacol. 163, 24-36 (2014).

90. Bolger, A. M., Lohse, M. \& Usadel, B. Trimmomatic: a flexible trimmer for Illumina sequence data. Bioinformatics 30, 2114-2120 (2014).

91. Li, H. \& Durbin, R. Fast and accurate short read alignment with Burrows-Wheeler transform. Bioinformatics 25, 1754-1760 (2009).

92. Ramirez, F. et al. High-resolution TADs reveal DNA sequences underlying genome organization in flies. Nat. Commun. 9, 189 (2018).

93. Lopez-Delisle, L. et al. pyGenomeTracks: reproducible plots for multivariate genomic data sets. Bioinformatics 37, 422-423 (2020).

94. Smit, A. F. A. \& Hubley, R. RepeatModeler Open-1.0 http://www. repeatmasker.org (Institute for Systems Biology) (2008).

95. Jurka, J. Repbase update: a database and an electronic journal of repetitive elements. Trends Genet. 16, 418-420 (2000).

96. Smit, A. F. A., Hubley, R. \& Green, P. RepeatMasker Open-4.0 http://www repeatmasker.org (Institute for Systems Biology) (2013).

97. Smith, C. D. et al. Improved repeat identification and masking in Dipterans. Gene 389, 1-9 (2007).

98. Altschul, S. F., Gish, W., Miller, W., Myers, E. W. \& Lipman, D. J. Basic local alignment search tool. J. Mol. Biol. 215, 403-410 (1990).

99. Slater, G. S. \& Birney, E. Automated generation of heuristics for biological sequence comparison. BMC Bioinformatics 6, 31 (2005).

100. Korf, I. Gene finding in novel genomes. BMC Bioinformatics 5, 59 (2004).

101. Stanke, M. \& Waack, S. Gene prediction with a hidden Markov model and a new intron submodel. Bioinformatics 19, ii215-ii225 (2003).

102. Bowman, M. J., Pulman, J. A., Liu, T. L. \& Childs, K. L. A modified GC-specific MAKER gene annotation method reveals improved and novel gene predictions of high and low GC content in Oryza sativa. BMC Bioinformatics 18, 522 (2017).

103. Bateman, A. et al. The Pfam Protein Families Database http://www.sanger. ac.uk/Software/Pfam/ (2000).

104. Eddy, S. R. Multiple alignment using hidden Markov models. Proc. Int. Conf. Intell. Syst. Mol. Biol. 3, 114-120 (1995).

105. Campbell, M. S., Holt, C., Moore, B. \& Yandell, M. Genome annotation and curation using MAKER and MAKER-P. Curr. Protoc. Bioinformatics 48, 4.11.1-4.11.39 (2014).

106. Vilella, A. J. et al. EnsemblCompara GeneTrees: complete, duplication-aware phylogenetic trees in vertebrates. Genome Res. 19, 327-335 (2009).

107. Ruan, J. et al. TreeFam: 2008 update. Nucleic Acids Res. 36, D735-D740 (2008)

108. Wallace, I. M., O’Sullivan, O., Higgins, D. G. \& Notredame, C. M-Coffee: combining multiple sequence alignment methods with T-Coffee. Nucleic Acids Res. 34, 1692-1699 (2006).

109. Sankoff, D., Deneault, M., Bryant, D., Lemieux, C. \& Turmel, M. in Comparative Genomics. Computational Biology Vol. 1 (eds Sankoff, D. \& Nadeau, J. H.) (Springer, 2000).

110. Sawa, G., Dicks, J. \& Roberts, I. N. Current approaches to whole genome phylogenetic analysis. Brief. Bioinform. 4, 63-74 (2003).

111. Farris, J. S. Phylogenetic analysis under Dollo's law. Syst. Biol. 26, 77-88 (1977).

112. Felsenstein, J. PHYLIP-phylogeny inference package (ver. 3.2). Cladistics 5 164-166 (1989).

113. Langmead, B. \& Salzberg, S. L. Fast gapped-read alignment with Bowtie 2. Nat. Methods 9, 357-359 (2012).

114. Li, H. et al. The Sequence Alignment/Map format and SAMtools. Bioinformatics 25, 2078-2079 (2009).

115. Thorvaldsdottir, H., Robinson, J. T. \& Mesirov, J. P. Integrative Genomics Viewer (IGV): high-performance genomics data visualization and exploration. Brief. Bioinform. 14, 178-192 (2013).

116. Ramírez, F., Dündar, F., Diehl, S., Grüning, B. A. \& Manke, T. DeepTools: a flexible platform for exploring deep-sequencing data. Nucleic Acids Res. 42, W187-W191 (2014)

117. Grabherr, M. G. et al. Full-length transcriptome assembly from RNA-seq data without a reference genome. Nat. Biotechnol. 29, 644-652 (2011).
118. Kopylova, E., Noé, L. \& Touzet, H. SortMeRNA: fast and accurate filtering of ribosomal RNAs in metatranscriptomic data. Bioinformatics 28, 3211-3217 (2012).

119. Fernandez-Minan, A., Bessa, J., Tena, J. J. \& Gomez-Skarmeta, J. L. Assay for transposase-accessible chromatin and circularized chromosome conformation capture, two methods to explore the regulatory landscapes of genes in zebrafish. Methods Cell Biol. 135, 413-430 (2016).

120. Gaspar, J. M. Improved peak-calling with MACS2. Preprint at bioRxiv https://doi.org/10.1101/496521 (2018).

121. Quinlan, A. R. \& Hall, I. M. BEDTools: a flexible suite of utilities for comparing genomic features. Bioinformatics 26, 841-842 (2010).

122. Babicki, S. et al. Heatmapper: web-enabled heat mapping for all. Nucleic Acids Res. 44, W147-W153 (2016).

123. Wickham, H. ggplot2: Elegant Graphics for Data Analysis 2nd edn (Springer Verlag, 2016).

124. Lufkin, T. In situ hybridization of whole-mount mouse embryos with RNA probes: preparation of embryos and probes. CSH Protoc. 2007, pdb.prot4822 (2007)

125. Lufkin, T. In situ hybridization of whole-mount mouse embryos with RNA probes: hybridization, washes, and histochemistry. CSH Protoc. 2007, pdb. prot4823 (2007).

126. Tatsumi, N. et al. Molecular developmental mechanism in polypterid fish provides insight into the origin of vertebrate lungs. Sci. Rep. 6, 30580 (2016).

127. Hara, Y. et al. Shark genomes provide insights into elasmobranch evolution and the origin of vertebrates. Nat. Ecol. Evol. 2, 1761-1771 (2018).

128. Komisarczuk, A. Z., Kawakami, K. \& Becker, T. S. Cis-regulation and chromosomal rearrangement of the $f g f 8$ locus after the teleost/tetrapod split. Dev. Biol. 336, 301-312 (2009).

129. Marinic, M., Aktas, T., Ruf, S. \& Spitz, F. An integrated holo-enhancer unit defines tissue and gene specificity of the Fgf8 regulatory landscape. Dev. Cell 24, 530-542 (2013).

130. Hornblad, A., Bastide, S., Langenfeld, K., Langa, F. \& Spitz, F. Dissection of the Fgf8 regulatory landscape by in vivo CRISPR-editing reveals extensive intra- and inter-enhancer redundancy. Nat. Commun. 12, 439 (2021).

\section{Acknowledgements}

We are thankful for grant support from NIH R01OD011116 (I.B.), NSF DDIG DEB1600920 (M.B.H.), NSF IOS-1755242 (A.D.) and NSF IOS-1755330 (J.A.Y.). Parts of this work have been supported by the Agence Nationale de la Recherche, France (ANR GenoFish project, 2016-2021, ANR-16-CE12-0035) to H.R.C., C.B., E.P., A.L. and Y.G. E.P., C.B. and H.R.C. received support under the program 'Investissements d'Avenir' launched by the French government and implemented by ANR with references ANR10-LABX-54 MEMOLIFE and ANR-10-IDEX-0001-02 PSL* Université Paris. We thank F. Feng for access to Oneida Lake bowfin spawning habitats, the Bauer Core at Harvard University for sequencing fin transcriptomes and M. Gundappa (FISH \& LINES) for species illustrations. Species silhouettes were obtained from http://PhyloPic.org. We are exceedingly grateful to the late J.L. Gómez-Skarmeta for his guidance on establishing ATAC-seq in holosteans.

\section{Author contributions}

A.W.T. and I.B. conceived the study and wrote the article with input from co-authors; I.B. coordinated genome sequencing in collaboration with Dovetail Genomics; E.F., A.R.M., Q.F., A.F. and S.R.D. provided bowfin samples for genomic, chromatin and transcriptomic analyses; A.W.T., K.L.C. and I.B. performed genome annotation; S.R.D., A.W.T., B.L.R. and I.B. sampled bowfin for sex-determination analyses performed by Q.P., R.F., M.M. and Y.G.; E.P., A.L., J.M., H.R.C., C.B., A.W.T. and I.B. analyzed genome evolution; A.W.T. and I.B. generated phylogenomic analyses; D.J.W., T.O., A.D. and J.A.Y. performed immunogenome analyses; K.K. analyzed SCPP genes; A.W.T., O.E.F. and I.B. performed gene-regulatory analyses; M.L., I.B., M.B.H. and M.P.H. analyzed Hox genes; M.B.H., M.P.H. and I.B. investigated fin development.

\section{Competing interests}

The authors declare no competing interests.

\section{Additional information}

Extended data is available for this paper at https://doi.org/10.1038/s41588-021-00914-y. Supplementary information The online version contains supplementary material available at https://doi.org/10.1038/s41588-021-00914-y.

Correspondence and requests for materials should be addressed to I.B.

Peer review information Nature Genetics thanks Kiyoshi Naruse, Yongxin Li, and the other, anonymous, reviewer(s) for their contribution to the peer review of this work.

Reprints and permissions information is available at www.nature.com/reprints. 

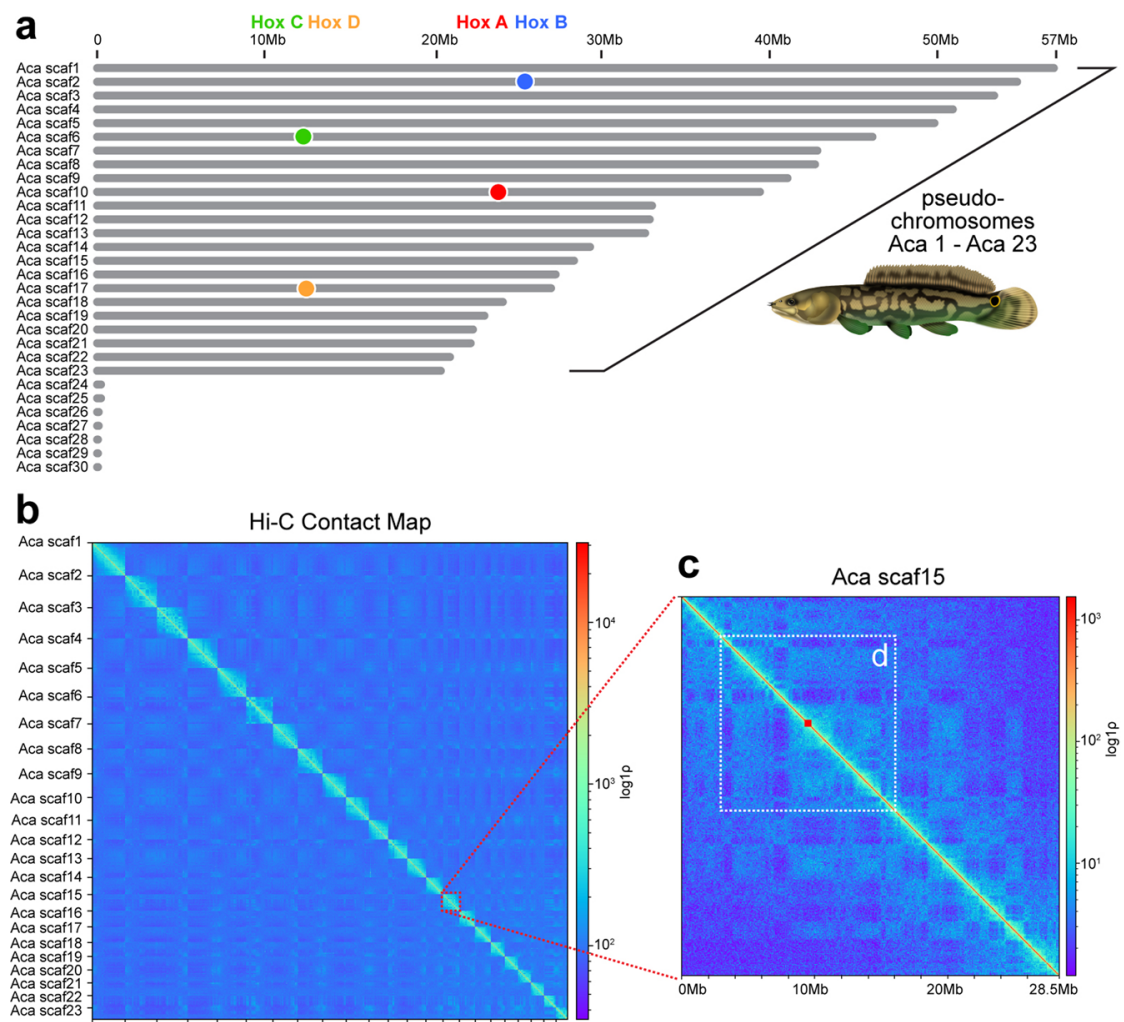

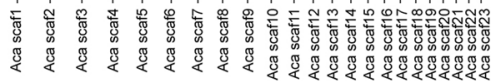

d Aca scaf15
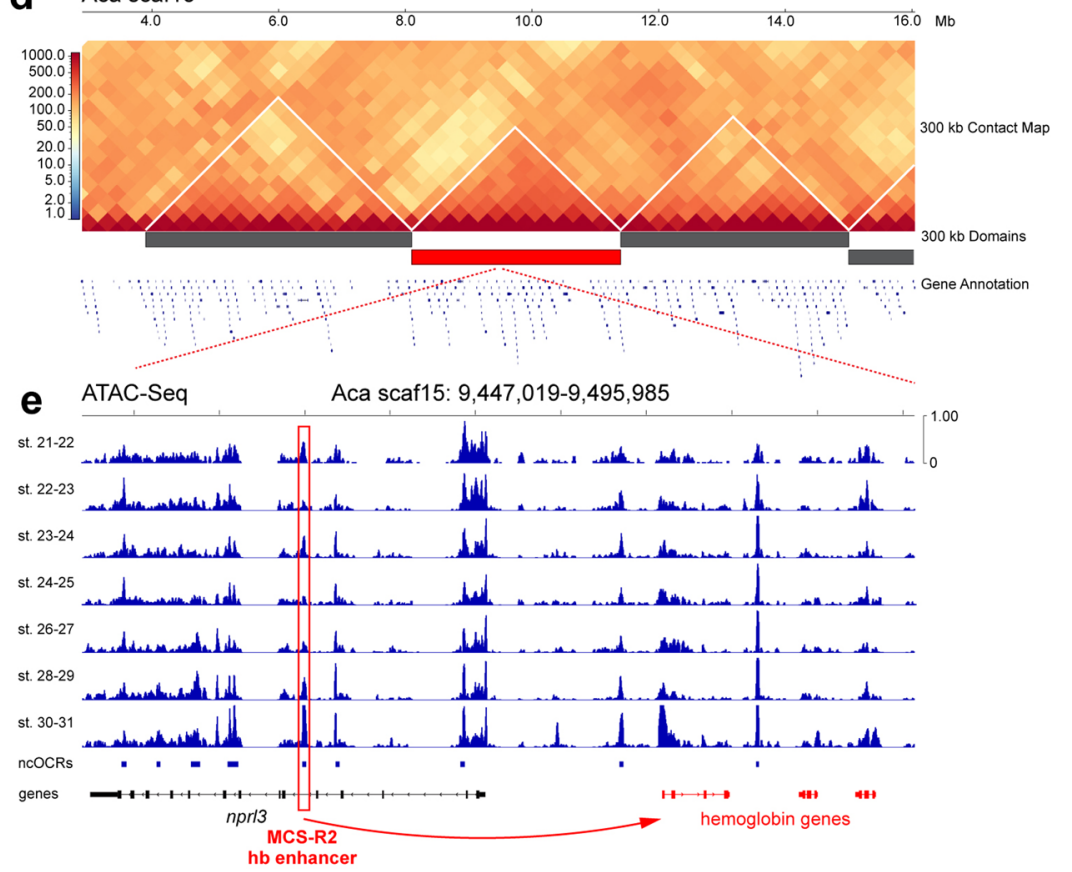

f Synteny

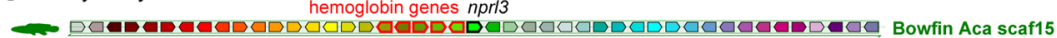

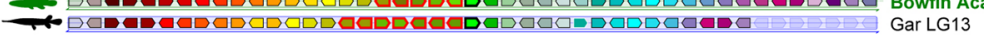

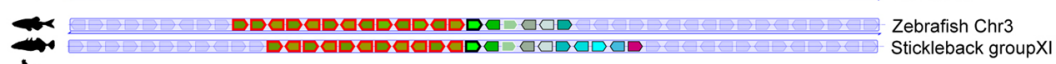

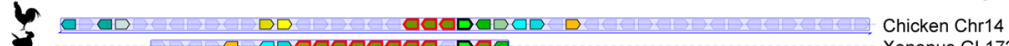

1.

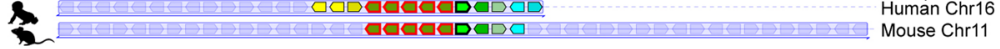


Extended Data Fig. 1| The bowfin genome. (a) Size distribution of the 30 largest scaffolds in the AmiCal1 assembly. Aca scaf1 to Aca scaf23 match the number of chromosomes in the bowfin genome ${ }^{21,29}$ and are thus considered pseudochromosomes. Locations of the four bowfin Hox gene clusters Hox A-D are indicated by colored circles. (b) Hi-C contact map for the 23 pseudochromosomes, generated from blood sample. Resolution: 500-kb bins. The red dashed box indicates Aca scaf15. (c) Hi-C contact map for Aca scaf15. Resolution: 50-kb bins. (d) Topologically Associating Domains (TADs) called using a resolution of 300-kb bin size in the region of Aca scaf15 indicated by the white dashed box in (c). (e) ATAC-Seq profile through bowfin development within the central TAD in (d). ATAC-Seq peaks and ncOCRs are observed for a putative bowfin ortholog of the MCS-R2 hb enhancer in intron 5 of the nprl3 gene known to regulate flanking hemoglobin genes ${ }^{62}$ (red). (f) Conserved synteny of the region containing hemoglobin genes (red highlighted arrows) on bowfin Aca scaf15 to gar, teleosts, and tetrapods, centered on the nprl3 gene (central green arrow) that contains the putative MCS-R2 intronic hb enhancer. 
a

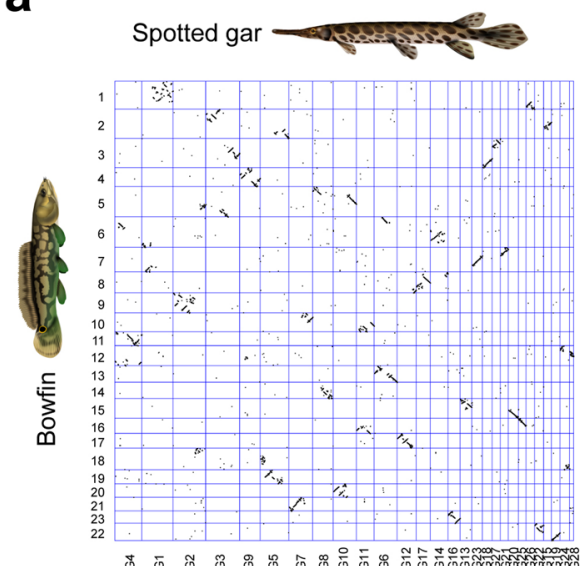

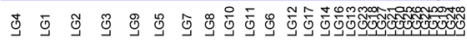

b

b

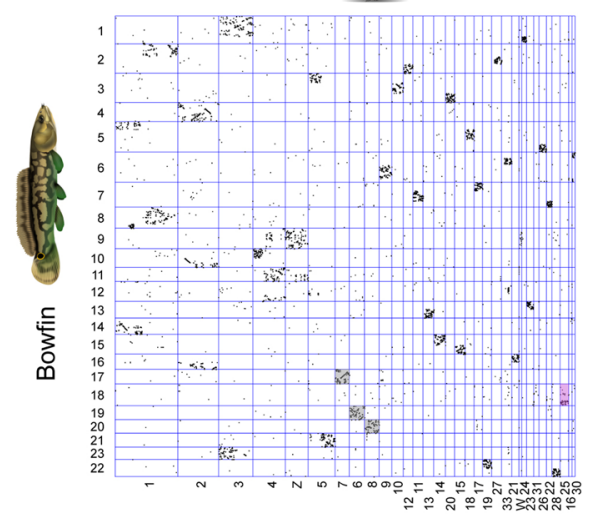

C

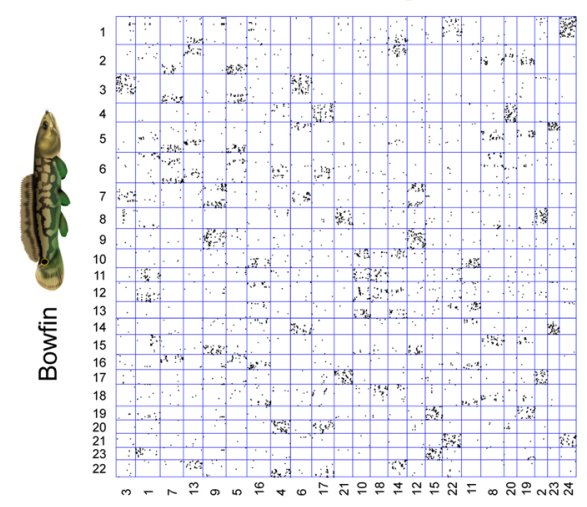

d
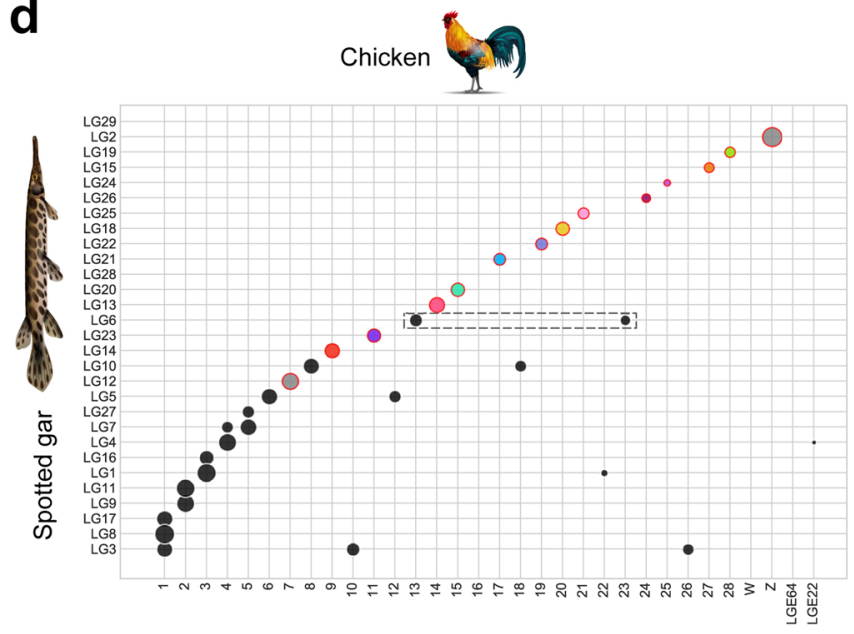

e
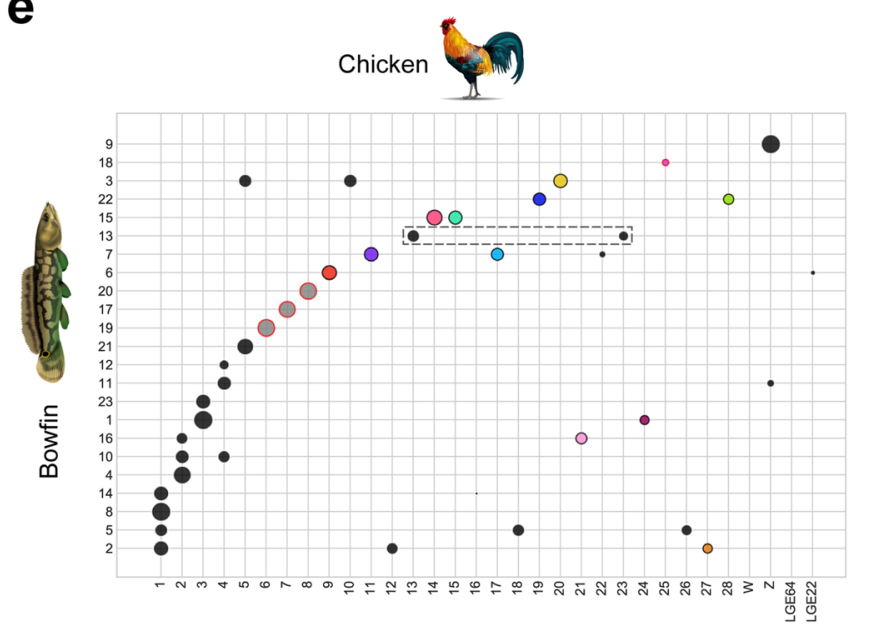

Holostean chromosome fusion

f

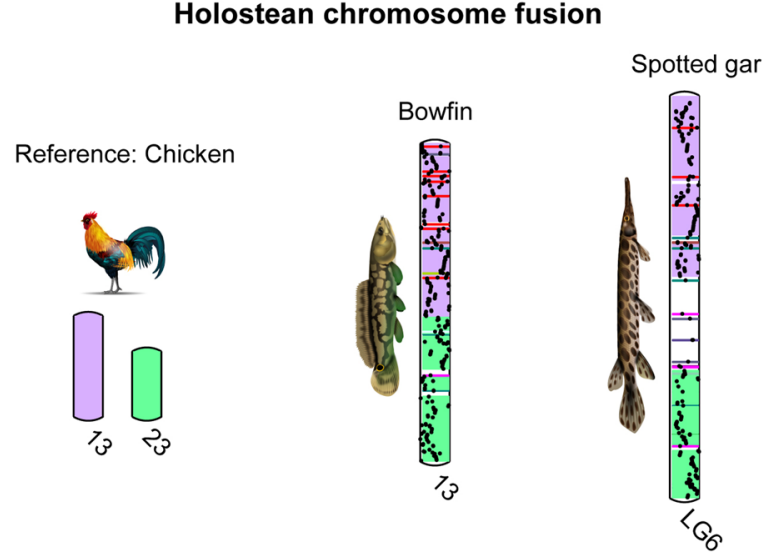

Bony vertebrate

ancestral

chromosomes

Macro-chromosomes

Z - LG2

7 - LG12

Micro-chromosomes

28 - LG19

27 - LG15

25 - LG24

24 - LG26

21 - LG25

20 - LG18

$19-$ LG22

17 - LG21

15 - LG20

14 - LG13

11 - LG23

9 - LG14

\# orthologs

- 50

500

Chromosomes 1-to-1 Chicken-Bowfin

Macro-chromosomes

- $6-19$

$7-17$

ए $8-20$

Micro-chromosomes

25-18

Extended Data Fig. 2 | Conserved synteny analyses. Oxford grid comparisons of bowfin vs. gar (a), chicken (b), and medaka (c) genomes. (d) Orthologies between the bowfin and chicken chromosomes. (e) Orthologies between the gar and chicken chromosomes. Circles represent the number of orthologous genes shared between pairs of chromosomes (if in excess compared to random expectations). One-to-one orthologous chromosome pairs are circled in red and summarized on the right. The dotted rectangle highlights a potential shared fusion in bowfin and gar. (f) Putative holostean-specific chromosome fusion. Bowfin chromosome 13 and spotted gar LG6 are painted according to chicken chromosomes 13 and 23. 


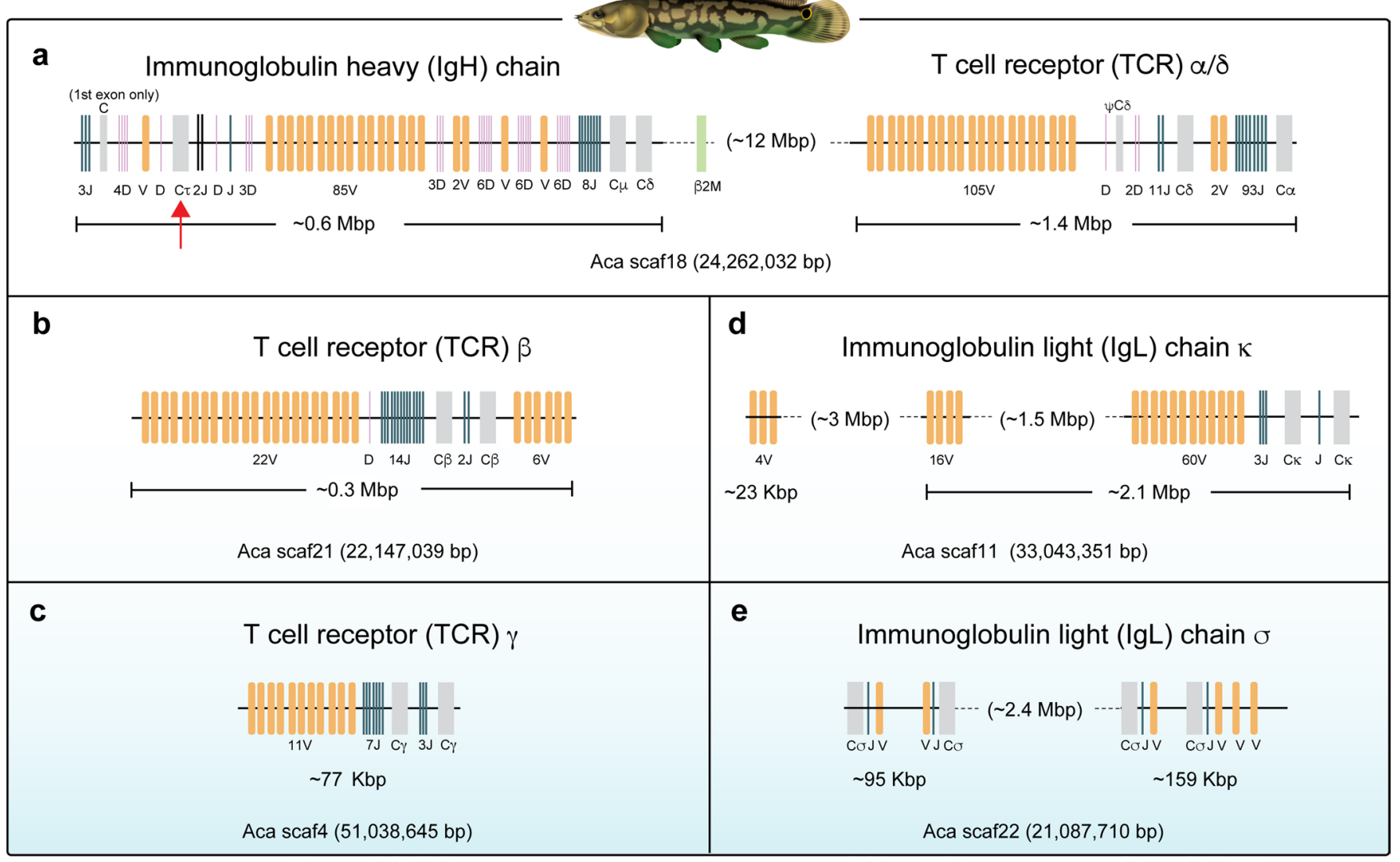

Extended Data Fig. 3 | Genomic organization of bowfin immunoglobulin and TCR genes. (a) The immunoglobulin heavy (IgH) chain and T cell receptor (TCR) $\alpha / \delta$ loci are separated by $12 \mathrm{Mb}$ on pseudochromosome 18 (Aca scaf18). The IgH locus encodes 3 classes of Ig constant domains, $\mathrm{C} \tau, \mathrm{C} \mu$ and $\mathrm{C} \delta$. The red arrow indicates $\mathrm{C} \tau$, which was previously thought to be teleost-specific. Note that the IgL sigma- 2 locus is present in the middle of $V$ gene segment cluster of the IgH locus (not shown). (b) The TCR $\beta$ locus is encoded on Aca scaf21. (c) The TCR locus is located on Aca scaf4. (d) The lg light $(\mathrm{IgL})$ chain kappa locus is present on Aca scaf11. (e) The IgL chain sigma loci are encoded on Aca scaf22. Multiple genes (including pseudogenes) other than Ig and TCR are present in these pseudochromosomes, but not shown. $V$ domains are shaded orange, $D$ segments purple, J segments blue, and constant (C) domains gray. 
a

Human Fetal Chromatin Accessibility (17:59,529,500-59,567,000; GRCh37)

0

Bowfin tbx4 (scaf22:4,672,549-4,699,496)

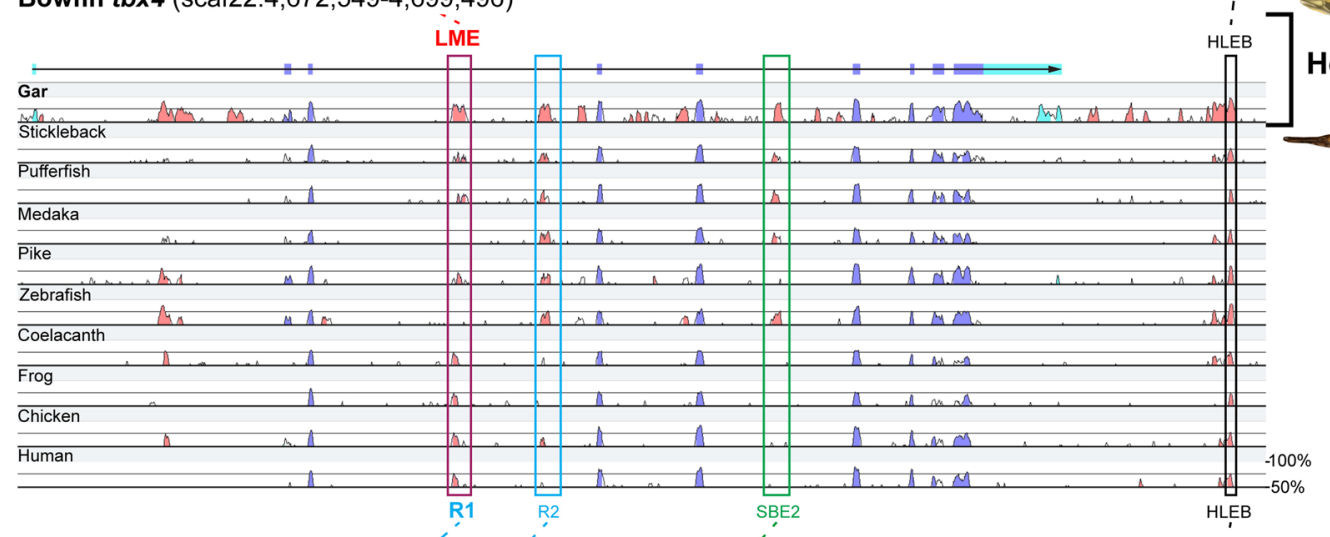

Stickleback tbx4 (groupl:18,564,510-18,584,211)

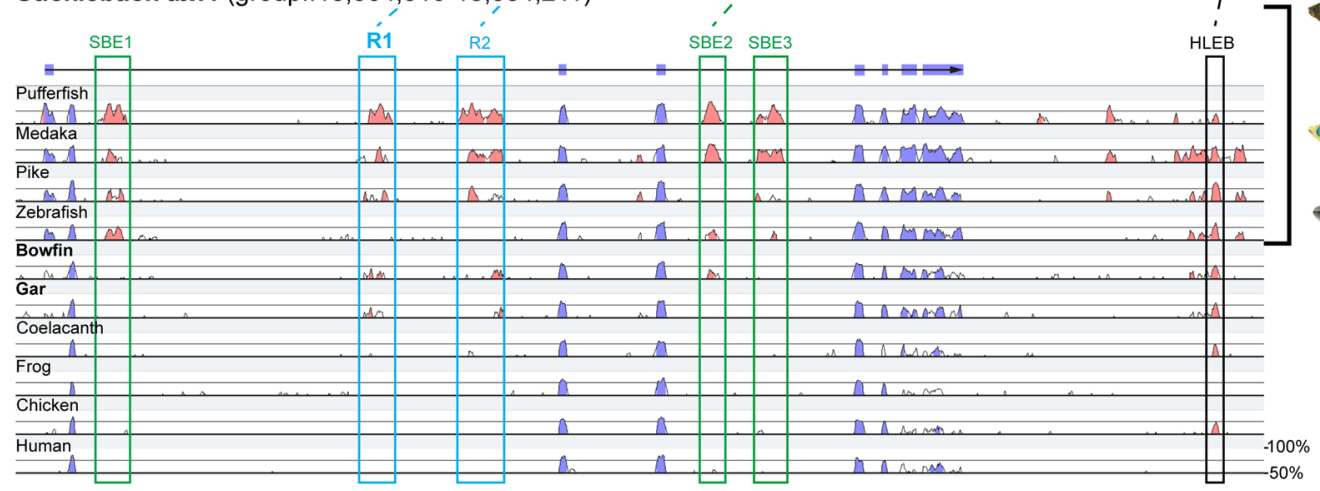

b $_{\text {Human Fetal Lung sci-RNA-Seq3:TBX4 }}$

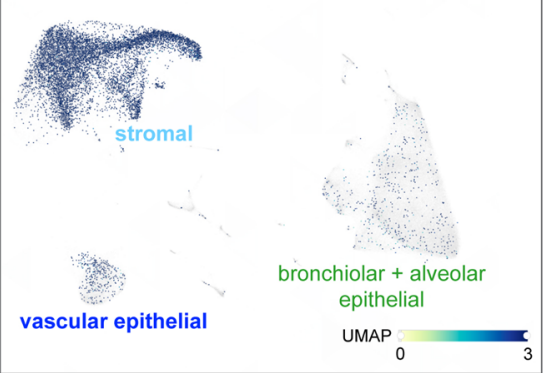


Extended Data Fig. 4 | The holostean bridge connects vertebrate Tbx4 gene enhancers. (a) Human fetal TBX4 chromatin accessibility shows a peak at the 'lung mesenchyme enhancer' (LME) in lung cells. (b) Human TBX4 single cell expression in fetal lung. See Supplementary Note 9 for details. (c) VISTA SLAGAN alignments with human (top), bowfin (middle), and stickleback (bottom) as reference. Top: Human TBX4 intron 3 'lung mesenchyme enhancer' (LME, red box), driving expression in the developing mammalian lung bud ${ }^{60,61}$, shows conservation with other tetrapods, coelacanth, bowfin, and gar (and also with bichir ${ }^{12,126}$ and some sharks ${ }^{127}$, not shown), but no conservation with teleosts. HLEB: TBX4 hind limb enhancer $B^{60}$ (black box). Bottom: The stickleback tbx4 gene contains three putative 'swimbladder enhancers'86 (SBE1-3, green boxes). SBE2 is conserved with bowfin but not lobefinned vertebrates. Intron 3 regions R1 and R2 (blue boxes) appear as conserved among neopterygians (teleosts, bowfin, gar), but not with coelacanth or tetrapods. HLEB (black box) is also present. Middle: The bowfin tbx4 gene identifies different conserved regions defined in the human- and sticklebackcentric alignments (top, bottom): SBE2 (green box), R2, and HLEB. Lobe-finned LME and neopterygian R1 elements overlap within the bowfin genome, and are thus orthologous conserved non-coding elements, connected via the holosteans bridge (right). This bowfin LME/R1 region is also a bowfin developmental ncOCR (main text Fig. 4f, where it is shown on the reverse strand). 
a

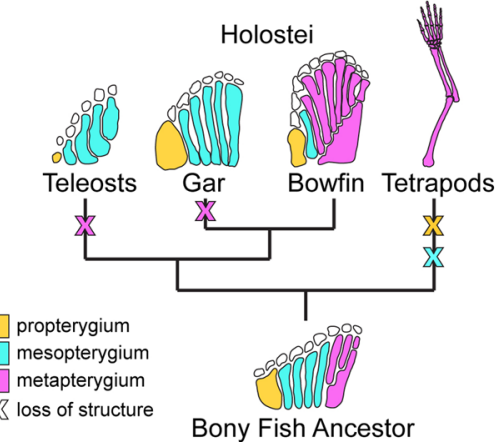

b

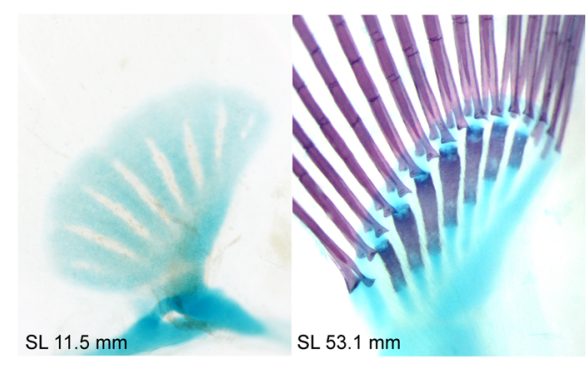

C

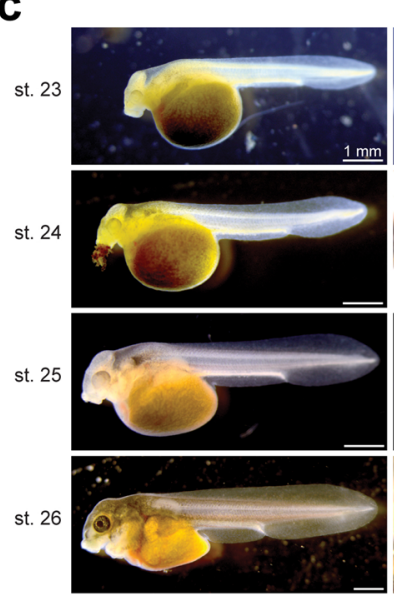

Dorsal

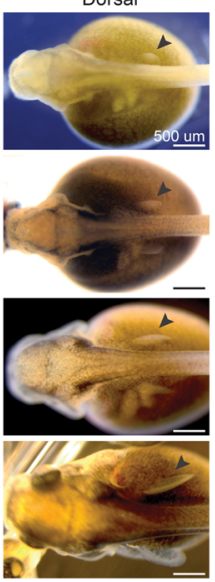

Lateral

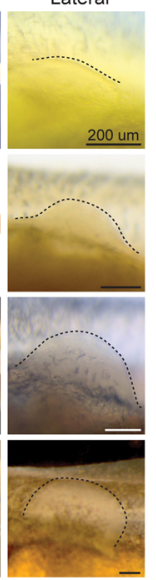

d

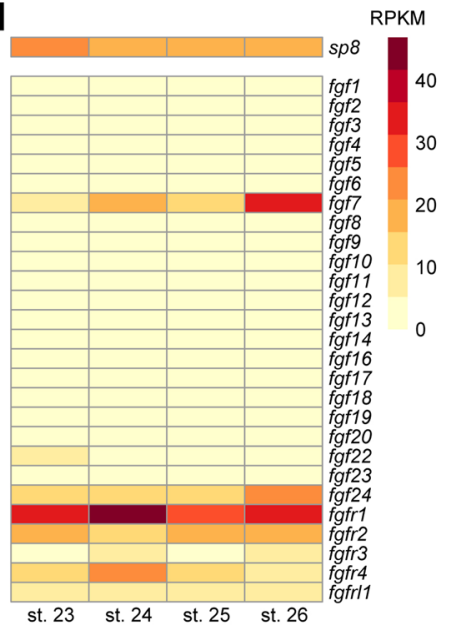

e

Reference:

Loc LG5:30,923,235-31,183,606

LoC

Aca 19

GacA

GacB IX

Mmu 19 (1)

CE39 CE40
22
btrc

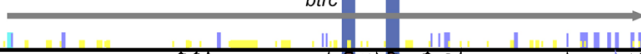

$s / c 2 a 15$

Loc

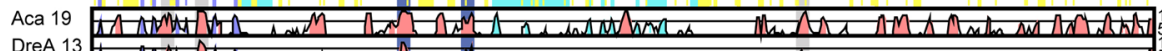

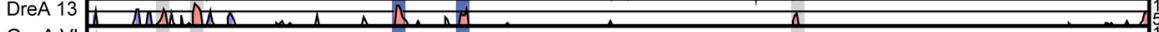

GacA

DreB 1

GacB IX

Mmu 19

II

III $\triangle \mathrm{A} \Lambda \mathrm{C}$

(1)

Loc

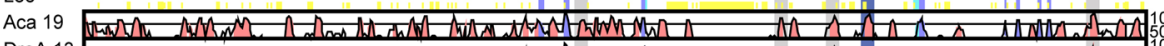

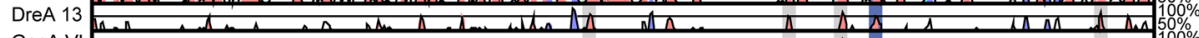

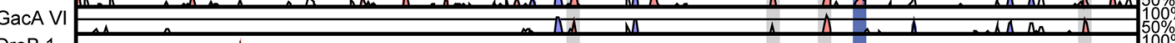

DreB 1

GacB IX

$\mathrm{Mmu} 19$

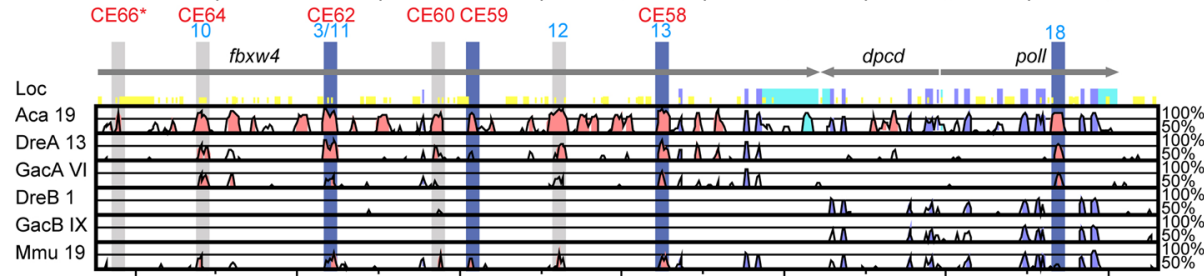

Extended Data Fig. 5 | See next page for caption. 
Extended Data Fig. 5 | Bowfin pectoral fin Evo-Devo. (a) Evolution of the pectoral endoskeleton in bony fishes. Elements are colored based on which portion of the fin they comprise. From the tribasal ancestral condition, teleosts and gars independently lost the metapterygium, while tetrapods lost the propterygium and mesopterygium. Bowfin is the closest living relative of the teleosts that retains the metapterygium. (b) Representative bowfin pectoral fins at two different developmental stages cleared and stained with alcian blue for cartilage and alizarin red for bone. Anterior to left, distal to

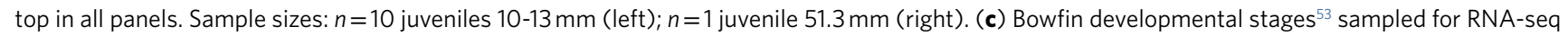
transcriptomics (representative of $n=20$ individuals per stage). Arrow in dorsal view (middle column) indicates developing pectoral fin, dotted line in lateral view (right column) indicates outline of the developing pectoral fin bud. (d) Pectoral fin RNA-Seq of sp8 and Fgf signaling genes. Expression levels in Reads Per Kilobase Million (RPKM). (e) Genomic alignment of bony vertebrate fgf8 regions. mVISTA plot showing sequence conservation of bowfin (Aca), zebrafish (Dre; fgf8a and fgf8b paralogons), stickleback (Gac; fgf8a and fgf8b paralogons), and mouse (Mmu) vs. spotted gar (Loc) as reference. Shaded areas highlight known regulatory elements for zebrafish (blue numbers) ${ }^{128}$ and mouse (red CE numbers) ${ }^{129}$; blue shade indicates bowfin ncOCRs (Supplementary Table 26). All relevant elements, including those important for expression in the apical ectodermal ridge of the mouse limb ${ }^{130}$, that is, CE58, CE59, CE66, CE80, except for CE61 (also absent from zebrafish), are found in gar and bowfin. CE66 (asterisk) was identified by sequence similarity to human. 


\section{Reporting Summary}

Nature Research wishes to improve the reproducibility of the work that we publish. This form provides structure for consistency and transparency in reporting. For further information on Nature Research policies, see our Editorial Policies and the Editorial Policy Checklist.

\section{Statistics}

For all statistical analyses, confirm that the following items are present in the figure legend, table legend, main text, or Methods section.

$\mathrm{n} / \mathrm{a}$ Confirmed

$\bigotimes$ The exact sample size $(n)$ for each experimental group/condition, given as a discrete number and unit of measurement

\ A statement on whether measurements were taken from distinct samples or whether the same sample was measured repeatedly

The statistical test(s) used AND whether they are one- or two-sided

Only common tests should be described solely by name; describe more complex techniques in the Methods section.

$\bigotimes$ A description of all covariates tested

$\square$ A description of any assumptions or corrections, such as tests of normality and adjustment for multiple comparisons

$\triangle$ A full description of the statistical parameters including central tendency (e.g. means) or other basic estimates (e.g. regression coefficient)

X AND variation (e.g. standard deviation) or associated estimates of uncertainty (e.g. confidence intervals)

For null hypothesis testing, the test statistic (e.g. $F, t, r$ ) with confidence intervals, effect sizes, degrees of freedom and $P$ value noted Give $P$ values as exact values whenever suitable.

\For Bayesian analysis, information on the choice of priors and Markov chain Monte Carlo settings

$\bigotimes \square$ For hierarchical and complex designs, identification of the appropriate level for tests and full reporting of outcomes

X| $\square$ Estimates of effect sizes (e.g. Cohen's $d$, Pearson's $r$ ), indicating how they were calculated

\section{Our web collection on statistics for biologists contains articles on many of the points above.}

\section{Software and code}

Policy information about availability of computer code

Data collection Software was only used for data analyses.

Data analysis Custom scripts and workflows for the Pool-Seq sex determination analysis are available at https://github.com/RomainFeron/papersexdetermination-bowfin, for the gene order phylogeny at https://github.com/DyogenlBENS/BowfinGOPhylogeny. Other software was used as described in detail in the Methods section. ATAC-Seq reads mapping to the mitochondrial genome were removed with removeChrom.py [available from https://github.com/harvardinformatics/ATAC-seq/blob/master/atacseq/removeChrom.py]. Other standard bioinformatic softwares were used and cited in the manuscript such as: Augustus v2.6.1, BEAST2 v2.6.3, BEDtools 2.27.1, BLAST, Bowtie v2.3.4.1, BUSCO v5, BWA v0.7.1718, CEGMA, CLC Genomics Workbench, ClustalW, Cufflinks, deepTools v3.5.1, Ensembl Compara, Exonerate v2.2.0, Galaxy, Geneious 9.1.8, HiRise, HMMER v3, HOMER v4.11, HiCExplorer v3.6, IGV v2.4.16, IQ-TREE, M-Coffee, MACS2 v2.2.6, MAKER v2.31, MEGAX, Meraculus, mVISTA, Orthofinder v1.1.3, Phylip, PicardTools v2.18.1, Progressive Cactus v.1.2.3, RAXML HPC-HYBRID-AVX, RepeatMasker v4.0.5, RepeatModeler v1.0.8, SAMtools v1.11, SNAP, SortmeRNA, TreeBeST, Trimmomatic v0.38, and Trinity v2.8.5.

For manuscripts utilizing custom algorithms or software that are central to the research but not yet described in published literature, software must be made available to editors and

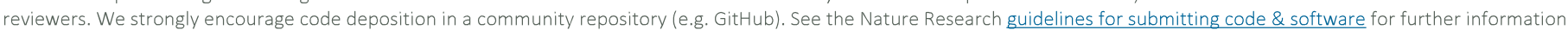


Policy information about availability of data

All manuscripts must include a data availability statement. This statement should provide the following information, where applicable:

- Accession codes, unique identifiers, or web links for publicly available datasets

- A list of figures that have associated raw data

- A description of any restrictions on data availability

The bowfin reference genome assembly (AmiCal1) is available at GenBank under the accession number PESF00000000, raw reads at the Sequence Read Archive under accession numbers SRR14766073-SRR14766075. Transcriptomic and ATAC-Seq reads are available under accession numbers SRP281665 and SRP252716; assembled transcripts are available under accession number GIOP00000000. The MAKER gene annotation is available at https://github.com/AndrewWT/ AmiaGenomics. Data for synteny analyses and the gene order phylogeny are available at https://github.com/DyogenIBENS/BowfinGOPhylogeny.

\section{Field-specific reporting}

Please select the one below that is the best fit for your research. If you are not sure, read the appropriate sections before making your selection.

Life sciences

Behavioural \& social sciences

Ecological, evolutionary \& environmental sciences

For a reference copy of the document with all sections, see nature.com/documents/nr-reporting-summary-flat.pdf

\section{Ecological, evolutionary \& environmental sciences study design}

All studies must disclose on these points even when the disclosure is negative.

Study description

Analyses consisted of standard bioinformatic pipelines. Detailed methods, and parameters and softwares used are described in the manuscript. When possible replicates were used to increase statistical power, and all data was confirmed to be of high quality before analyses.

Research sample

Both bowfin (Amia calva) and spotted gar (Lepisosteus oculatus) are not threatened. Samples were chosen from habitats where they are abundant and accessible. Samples were collected with the necessary permits and represent the Louisiana (adult bowfin, embryonic spotted gar) and Oneida Lake, NY (embryonic/larval bowfin) populations as described in the manuscript. Adult bowfin are sexually dimorphic and sex was determined after sacrifice upon dissection of gonads. Bowfin and gar developmental staging was performed by comparison of their morphology to that described in developmental staging literature.

Sampling strategy Sampling procedures and sample sizes for individual experiments are provided in the Methods section. To obtain sufficient material, pooling of embryos and tissues was necessary for developmental ATAC-Seq and RNA-Seq experiments in a stage-specific, empirically determined manner as detailed in the Methods section. When possible, replicates were sequenced for developmental genetics. Data quality was assessed and determined to be of high quality before and after analyses as described in the manuscript.

Data collection

All data was collected in the laboratories of the authors or downloaded from repositories like NCBI Genbank, Ensembl, and the VISTA enhancer browser as well as the literature as described in the manuscript. Sequencing instruments used for the generation sequence data include Illumina HiSeq X Ten, Illumina NextSeq v2.5, and Illumina NovaSeq 6000 as detailed in the Methods section.

Timing and spatial scale All developmental gene expression and chromatin analyses were performed on the same population of bowfin in Oneida Lake NY, Apr-Mar 2016 and Apr-Mar 2018 during the natural spawning season. Sample collection was stopped when the last desired developmental state of a sample was reached. All adult bowfin were collected around Thibodaux, Louisiana in Feb 2015, Apr 2017, and May 2018. Gar embryos were collected from a laboratory spawn in Apr 2018 as described in the manuscript.

Data exclusions No data collected were excluded from analyses.

Reproducibility A single reference genome assembly from a single adult male bowfin was generated. A single reference immune transcriptome was generated from a single adult female bowfin. Pool-Seq was performed with 30 males and 30 females. Developmental ATAC-Seq experiments were performed in 1-2 replicates per stage, developmental RNA-Seq experiments in 1-3 replicates per stage as detailed in the Methods section. RNA in situ hybridization was performed with 10 replicates per gene and per species as detailed in the Methods section. Photos of embryos are representative of at least 5 individuals per developmental stage. See Methods and figure legends for further details.

Randomization Random samples were taken from pools of healthy adults and embryonic/larval clutches. To remove batch effects in sequence data, developmental ATAC and RNA reads were pooled into a single Illumina lane and normalized for comparisons to remove any technical differences in read depth.

Blinding

No blinding was necessary as all data were used for the genome assembly and sequence data generation and analyses thereof.

Did the study involve field work? \Yes $\square$ No 
Field conditions

Location

Access \& import/export

Disturbance
Animals were collected during spring March-May in both Lousiana and New York, when temperatures were warming prior to extensive annual habitat flooding. Bowfin and gar spawn during this time.

Atchafalaya Basin $\left(29^{\circ} 48^{\prime} 45.4^{\prime \prime} \mathrm{N} 91^{\circ} 13^{\prime} 14.9^{\prime \prime} \mathrm{W}\right)$ and Bayou Chevreuil $\left(29^{\circ} 54^{\prime} 50.6^{\prime \prime} \mathrm{N} 90^{\circ} 47^{\prime} 56.9^{\prime \prime} \mathrm{W}\right)$ in Louisiana, and Oneida Lake,

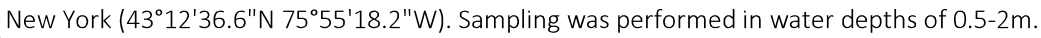

All animals were sampled in accordance with permits from Nicholls State University (\#IA046/IA053), Michigan State University (\#10/16-179-00), and Cornell University (\#2006-0013).

\section{Reporting for specific materials, systems and methods}

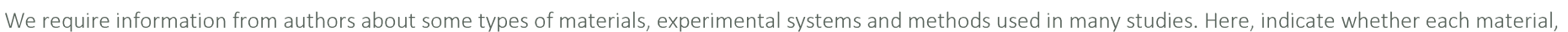

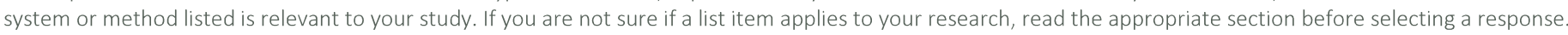

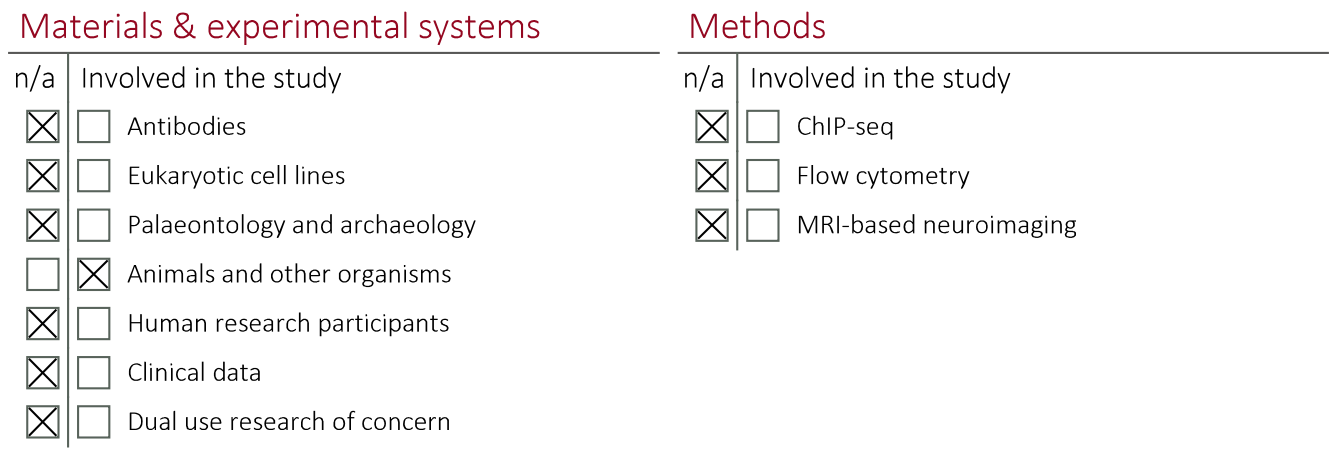

\section{Animals and other organisms}

Policy information about studies involving animals; ARRIVE guidelines recommended for reporting animal research

Laboratory animals

Wild animals

Field-collected samples

Ethics oversight

\begin{abstract}
Embryos and larvae of Amia calva (bowfin) and Lepisosteus oculatus (spotted gar) were used as laboratory animals. Lab animals were humanely euthanized according to Institutional Animal Care and Use Committee (IACUC) protocols from Nicholls State University (\#IA046/IA053), Michigan State University (\#10/16-179-00), and Cornell University (\#2006-0013).

Adult bowfin were captured in the Atchafalaya Basin and Bayou Chevreuil, Louisiana by electrofishing and transported to the laboratory in bayou water. DNA for the genome sample was taken from a sacrificed single adult male. Immune tissues (gill, spleen, intestine) were dissected from a sacrificed single adult female bowfin. For Pool-Seq analyses, fin clips were taken from 30 adult male and 30 adult female bowfins that were euthanized to allow for gonadal inspection by dissection. All animals were handled and euthanized according to Institutional Animal Care and Use Committee (IACUC) protocols from Nicholls State University (\#IA046/ IA053), Michigan State University (\#10/16-179-00), and Cornell University (\#2006-0013).
\end{abstract}

Bowfin embryos were collected from nests in Oneida Lake, New York. Eggs attached to nest material were collected with hand nets and transported to the laboratory in lake water with methylene blue added to abate fungal growth. In the laboratory, eggs were separated from nesting material by hand and placed in fresh lake water. Eggs and embryos were raised in static containers of lake water (16-18 degree Celsius) with a $12 \mathrm{~h}$ dark/12h light photoperiod and moved to fresh water every other day. Embryos and larvae were sampled at the relevant stages following the bowfin staging series by Ballard (1986). Adult bowfin were captured in the Atchafalaya Basin and Bayou Chevreuil, Louisiana. At the end of experiments, all animals were euthanized following the approved IACUC protocols from Nicholls State University (\#IA046/IA053), Michigan State University (\#10/16-179-00), and Cornell University (\#2006-0013).

\footnotetext{
Bowfin work was approved under Institutional Animal Care and Use Committee (IACUC) protocols from Nicholls State University (\#IA046/IA053), Michigan State University (\#10/16-179-00), and Cornell University (\#2006-0013). Bowfins for genome sequencing sex determination analysis, and immune transcriptomics were sampled in the Atchafalaya Basin and Bayou Chevreuil, Louisiana (USA). Bowfin embryos and larvae for developmental and fin bud transcriptomics, ATAC-Seq, and RNA in situ hybridization were collected from nests in Oneida Lake (New York, USA), then raised in the laboratory as described until sampling at the desired developmental stages. Spotted gar embryos were obtained, raised, and fixed as described and approved by the Nicholls State University (\#IA053) and the Michigan State University (\#10/16-179-00) IACUC.
} 